The Astrophysical Journal, 682:803-820, 2008 August 1

(C) 2008. The American Astronomical Society. All rights reserved. Printed in U.S.A.

\title{
WIDE-FIELD CHANDRA X-RAY OBSERVATIONS OF ACTIVE GALACTIC NUCLEI IN ABELL 85 AND ABELL 754
}

\author{
Gregory R. Sivakoff, ${ }^{1}$ Paul Martini, ${ }^{1}$ Ann I. Zabludoff, ${ }^{2}$ Daniel D. Kelson, ${ }^{3}$ and John S. Mulchaey ${ }^{3}$ \\ Received 2008 February 22; accepted 2008 April 24
}

\begin{abstract}
To better understand the mechanism or mechanisms that lead to AGN activity today, we measure the X-ray AGN fraction in a new sample of nearby clusters and examine how it varies with galaxy properties, projected clustercentric radius, and cluster velocity dispersion. We present new wide-field Chandra X-Ray Observatory observations of Abell 85, Abell 754, and the background cluster Abell 89B out to their virial radii. Out of $17 \mathrm{X}$-ray sources associated with galaxies in these clusters, we classify 7 as X-ray AGNs with $L_{\mathrm{X}, B}>10^{41} \mathrm{ergs} \mathrm{s}^{-1}$. Only 2 of these would be classified as AGNs based on their optical spectra. We combine these observations with archival data to create a sample of X-ray AGN from $6 z<0.08$ clusters and find that $3.4_{-0.8}^{+1.1} \%$ of $M_{R}<-20$ galaxies host X-ray AGNs with $L_{\mathrm{X}, B}>10^{41} \mathrm{ergs} \mathrm{s}^{-1}$. We find that more X-ray AGNs are detected in more luminous galaxies and attribute this to larger spheroids in more luminous galaxies and increased sensitivity to lower Eddington-rate accretion from black holes in those spheroids. At a given X-ray luminosity limit, more massive black holes can be accreting less efficiently, yet still be detected. If interactions between galaxies are the principal drivers of AGN activity, then the AGN fraction should be higher in lower velocity dispersion clusters and the outskirts of clusters. However, the tendency of the most massive and early-type galaxies to lie in the centers of the richest clusters could dilute such trends. While we find no variation in the AGN fraction with projected clustercentric radius, we do find that the AGN fraction increases significantly, from $2.6_{-0.8}^{+1.0} \%$ in rich clusters to $10.0_{-4.3}^{+6.2} \%$ in those with lower velocity dispersions.
\end{abstract}

Subject headings: galaxies: active — galaxies: clusters: general — galaxies: general — X-rays: galaxies — $\mathrm{X}$-rays: galaxies: clusters - X-rays: general

\section{INTRODUCTION}

What is the principal driver of active galactic nuclei (AGNs) in the nearby universe? Major mergers between gas-rich galaxies are largely accepted as the dominant fueling mechanism (e.g., Barnes \& Hernquist 1992) for the luminous quasar population. Under this fueling scenario, higher AGN fractions are predicted for environments in which gas-rich galaxies are likely to interact with one another. Galaxy harassment due to frequent high-speed fly-by interactions has also been invoked as a mechanism for fueling AGNs, as it drives dynamical instabilities that efficiently channel gas to the centers of galaxies (Moore et al. 1996; Lake et al. 1998). The strength of galaxy harassment does not necessarily peak in the center of clusters (Lake et al. 1998) and may be relatively constant within a cluster ( Treu et al. 2003); however, AGN fueling by galaxy harassment requires that the harassed galaxy contain a gas reservoir. Therefore, if galaxy harassment fuels AGNs, higher AGN fractions might also be expected in environments in which gas-rich galaxies are likely to interact with one another. Although galaxy densities are high, such interactions are not favored in the centers of rich clusters, whose galaxies are less (cold) gas-rich than their counterparts in the field (e.g., Giovanelli \& Haynes 1985) and where the large relative velocities between galaxies inhibits actual mergers. Higher fractions of AGNs are expected for lower velocity dispersion structures. The AGN fraction at the outskirts of clusters should also be larger as a higher fraction of gas-rich galaxies are found toward the outskirts of clusters and infalling structures with lower velocity dispersions may not yet

\footnotetext{
1 Department of Astronomy, 4055 McPherson Laboratory, Ohio State University, 140 West 18th Avenue, Columbus, OH 43210-1173; sivakoff@astronomy.ohiostate.edu.

${ }^{2}$ Steward Observatory, University of Arizona, 933 North Cherry Avenue, Room N204, Tucson, AZ 85721-0065.

${ }^{3}$ Carnegie Observatories, 813 Santa Barbara Street, Pasadena, CA 91101-1292.
}

have virialized. Some of this picture has been supported by numerous studies of clusters that identified AGNs by their optical spectra observed a substantial decrease in the number of cluster AGNs relative to the field (Gisler 1978). Specifically, Dressler et al. (1985) measured a decrease from 5\% to $1 \%$ in AGNs residing in bright galaxies.

On the other hand, a large fraction of elliptical galaxies $(\sim 35 \%-45 \%)$ contain low-ionization nuclear emission-line regions (LINERs; Ho et al. 1997), many of which may be ionized by the accretion disk of a low-luminosity AGN (Ho et al. 1993). These elliptical galaxies comprise a higher fraction of the galaxy populations in the high surface density regions at the centers of clusters (Dressler 1980). In particular, the most luminous elliptical galaxies $\left(M_{R}<-22\right)$ are much more centrally concentrated ( Thomas \& Katgert 2006). Toward the outskirts of clusters, progressively higher fractions of poststarburst and star-forming galaxies are found (e.g., Dressler 1980; Fisher et al. 1998). Therefore, a relation between AGNs and early-type galaxies could dilute or even reverse the trends predicted by gas-rich mergers or galaxy harassment.

To gain leverage on these issues, it is critical to improve on the range of environments probed by past studies. We are continuing a program that measures the AGN fraction with environment, probing cluster environments for these indirect signatures of AGN fueling mechanisms. To identify the AGNs we use X-ray observations. Galaxy studies in the nearby universe (e.g., Grimm et al. 2003; Kim \& Fabbiano 2004; Sun et al. 2007) indicate that contributions from the other potential sources of luminous X-ray emission besides an AGN, namely, X-ray binaries and the hot interstellar medium (ISM), only exceed X-ray luminosities of $\sim 10^{41} \mathrm{ergs} \mathrm{s}^{-1}$ for the most massive or massively star-forming galaxies. Thus, AGNs can be identified down to relatively low X-ray luminosities by considering galactic parameters such as their optical luminosity and star formation rate (SFR). In addition, X-ray 
observations can identify AGNs that lack obvious spectral signatures in visible wavelength spectra. Such signatures could potentially be absent due to selection effects (e.g., optical dilution of low-luminosity AGNs; Martini et al. 2002; Moran et al. 2002), obscuration (e.g., Matt 2002), or different accretion modes (e.g., radiatively inefficient accretion flows that do not produce emission lines; Yuan \& Narayan 2004).

In the most detailed study of X-ray AGNs in clusters to date, Martini et al. (2006, hereafter M06) studied eight lowredshift $(0.06<z<0.31)$ galaxy clusters and found that $\sim 5 \%$ of bright $\left(M_{R}<-20\right)$ cluster galaxies contain AGNs with $L_{\mathrm{X}, B}>$ $10^{41} \mathrm{ergs} \mathrm{s}^{-1}$, where $L_{\mathrm{X}, B}$ is the broadband $(0.3-8 \mathrm{keV}) \mathrm{X}$-ray luminosity. Most of these X-ray-identified AGNs lacked obvious AGN spectral signatures in visible wavelength spectra. In this sample the $L_{\mathrm{X}, B}>10^{42} \mathrm{ergs} \mathrm{s}^{-1} \mathrm{X}$-ray AGNs were centrally concentrated (Martini et al. 2007, hereafter M07). When fainter X-ray AGNs, with $L_{\mathrm{X}, B}>10^{41} \mathrm{ergs} \mathrm{s}^{-1}$, were included, no central concentration was found, although they had limited sensitivity to radial variations in their more distant clusters. While the increase in AGN fraction of bright X-ray AGNs is more consistent with the increased concentration of bright elliptical galaxies outlined above, as opposed to predictions from galaxy interactions, it is somewhat surprising under such a model that the lower luminosity AGNs are not also centrally concentrated.

While X-ray AGN fractions in nearby clusters have been previously measured, past observations have concentrated on the cores of clusters. In M07, $90 \%$ of the galaxies were within $0.5 r_{200}$, where $r_{200}$ is the physical radius within which the mean density of a virialized cluster exceeds the critical density at that redshift by a factor of 200 . The outskirts of clusters, and their different environment, are relatively unexplored. This highlights the value of X-ray observations that can identify X-ray AGNs beyond the cores of clusters. Nearby clusters allow the most sensitive measurements at both visible and X-ray wavelengths. But such observations must be made over wide fields of view (FOVs) to cover the entire cluster. With its superb spatial resolution, the Chandra $X$-Ray Observatory is ideal for detecting a central AGN; however, its widest FOV (using the ACIS-I detectors) is only $\sim 17^{\prime} \times$ $17^{\prime}$. For nearby clusters, this does not provide adequate coverage out to $r_{200}$.

To attain the best measurements on the radial distribution of AGNs for comparison to the opposing predictions, we undertook wide-field Chandra observations of two $z \sim 0.06$ clusters, Abell 85 and Abell 754. In X-rays, both Abell 85 (Kempner et al. 2002; Durret et al. 2005) and Abell 754 (e.g., Markevitch et al. 2003) show evidence of recent mergers of multiple components; both clusters show evidence of cold fronts in their intracluster medium (ICM). In particular, Abell 754 is often used as a prototype of a major cluster-cluster merger, with the peak of its X-ray emission well offset from the major galaxy clumps identified by optical data (Zabludoff \& Zaritsky 1995), while there is no such offset in Abell 85, where smaller structures appear to be falling on to the major component of Abell 85 (Durret et al. 1998). Both clusters already have detailed optical spectroscopy (Christlein \& Zabludoff 2003, hereafter CZ03) that established cluster membership and measured other spectral properties. We present the analysis of these observations in $\S 2$. We add these clusters and Abell 89B, an additional cluster in the Abell $85 \mathrm{FOV}$, to three clusters from the M06 study to form a sample of $z \lesssim 0.08$ clusters in $\S 3$. In $\S 4$ we detail the identification of sources as X-ray AGNs and spectroscopically identified AGNs, and compare their properties (photometric and radial distribution) to the underlying cluster population. We present the dependence of AGN fraction on velocity dispersion and redshift in $\S 5$. Finally, we discuss our conclusions in $\S 6$. All errors presented indicate the double-sided $1 \sigma$ confidence interval. ${ }^{4}$ Throughout this paper we assume that the cosmological parameters are $\left(\Omega_{M}, \Omega_{\Lambda}, h\right)=(0.3,0.7,0.7)$, where $H_{0}=100 \mathrm{hm} \mathrm{s}^{-1} \mathrm{Mpc}^{-1}$. All absolute magnitudes and luminosities are presented in their rest frame.

\section{CHANDRA OBSERVATIONS}

\subsection{Data Reduction}

For both Abell 85 (Fig. 1) and Abell 754 (Fig. 2), our widefield Chandra ACIS-I observations consist of a $\sim 40 \mathrm{ks}$ central archival field flanked by eight new $\sim 10$ ks fields. We list these observations in Table 1.

We reduced all data as uniformly as possible using CIAO $3.4^{5}$ with CALDB 3.3.0.1 and NASA's FTOOLS 6.0. ${ }^{6}$ Since these observations represent a combination of archival and new observations spanning over 6 years, there were minor differences in their reduction. For observations 0577, 0944, and 4881-4888, the frame times were $3.2 \mathrm{~s}$, while for observations 6793-6800, the frame times were $3.1 \mathrm{~s}$. Both observation 0577 and 0944 were telemetered and cleaned in faint mode. The new observations were telemetered and cleaned in very-faint mode, which leads to a reduced background. Observation 0577 was operated at $-110^{\circ} \mathrm{C}$, while the remaining observations were operated at $-120^{\circ} \mathrm{C}$. Thus, for observation 0577 , no corrections were made for time dependence of the gain or the charge-transfer inefficiency and photon energies were determined using the gain file acisD1999-09-16gainN0005.fits. The other observations were all corrected for the time dependence of the gain and the charge-transfer inefficiency with their photon energies determined using the gain file acisD2000-01-29gain _ ctiN0006.fits. For observation 0577 and 0944, we recreated bad pixel files using the newest tools to detect hot pixels and cosmicray afterglows. For all observations, we only consider events with ASCA grades of $0,2,3,4$, and 6 detected by ACIS-I. Known aspect offsets were applied for each observation. All observations were corrected for quantum efficiency degradation and had exposure maps determined at $1.5 \mathrm{keV}$. We excluded bad pixels, bad columns, and columns adjacent to bad columns or chip node boundaries.

Since we use local backgrounds and small extraction regions to analyze point sources, this analysis is not very sensitive to the periods of high background ("background flares") that Chandra may encounter. To avoid periods with extreme flaring, we excluded times for which the blank-sky rate was more than 3 times the expected blank-sky rate derived from calibrated blank-sky backgrounds. We only removed $\sim 14 \mathrm{ks}$ from observation 0507 . Final flare-filtered live exposure times for the five observations are listed in Table 1.

In Figures 1 and 2 we display the adaptively smoothed, exposure-corrected Chandra X-ray image of both fields using a minimum signal-to-noise ratio $(\mathrm{S} / \mathrm{N})$ per smoothing beam of 3 . The FOVs of the individual observations are overlaid. Both clusters have ICM in the central archival field; however, only a little diffuse gas extends into the flanking fields. There are point sources seen in these images; however, most are unassociated with the clusters. On these figures we also display the radii corresponding to $1 \mathrm{Mpc}$ and $r_{200}$. Abell 85 has nearly complete coverage to $1 \mathrm{Mpc}$ and partial coverage out to just beyond $r_{200}$. Although the coverage for Abell 754 is similar, there are more holes due to the unmatched roll angles of the observations. The Abell 85 fields

\footnotetext{
4 We note that previous error bars on the AGN fraction presented single-sided $90 \%$ confidence intervals, which are slightly larger (M06, M07).

5 See http://asc.harvard.edu/ciao.

$6 \mathrm{See}$ http:// heasarc.gsfc.nasa.gov/docs/software/lheasoft.
} 


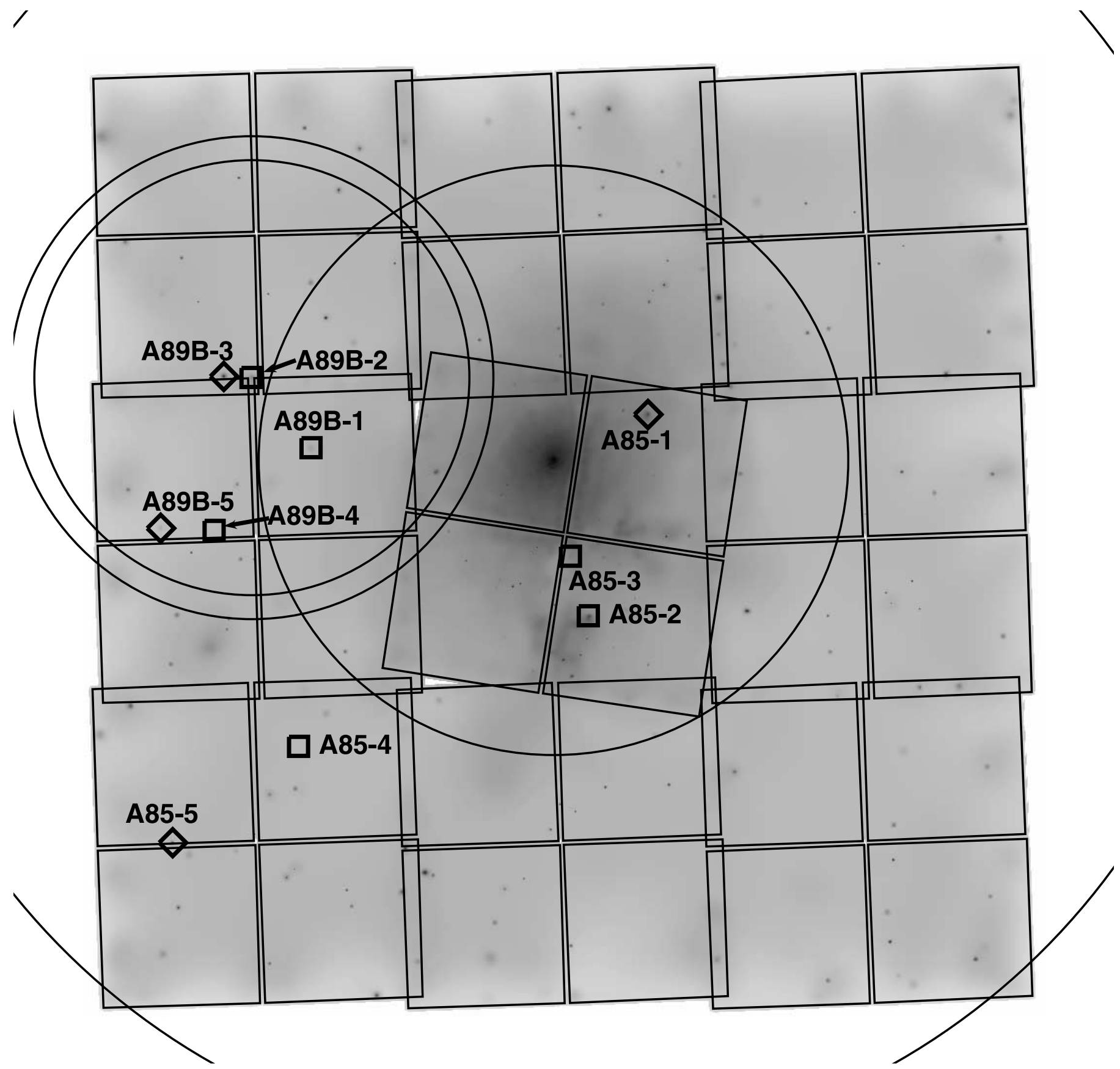

FIG. 1.-Adaptively smoothed Chandra mosaic of Abell 85 with individual Chandra FOVs indicated. An arcsinh scaling has been applied to bring out both point sources and intracluster gas. Inner and outer circles are used to display the $1 \mathrm{Mpc}$ radius and $r_{200}$, respectively, for both the Abell 85 (center) and Abell $89 \mathrm{~B}$ (east). Diamonds indicate galaxies detected as X-ray AGNs, while squares indicate X-ray-detected galaxies that are not considered X-ray AGNs.

also provide coverage for two other nearby large-scale structures, Abell 89B and Abell 89C (Durret et al. 1998). We include Abell 89B in this analysis, the less distant and richer of the two structures. Our Chandra data covers most of Abell 89B to its $r_{200}$ radius (Figure 3). Abell $89 \mathrm{C}$ is not included as our sample of $M_{R}<-20$ galaxies is incomplete at its redshift $(z \sim 0.096)$ and we were unable to self-consistently identify group members using the redshift and positions of candidate members $(\operatorname{see} \S 3)$.

\subsection{Source Detection and Analysis}

For each observation, we applied the CIAO wavelet detection algorithm (wavdetect) with scales ranging from 1 to 64 pixels in steps of $\sqrt{2}$ factors, requiring a source detection threshold of
$10^{-6}$ to identify discrete X-ray sources that are potential X-ray AGNs in these clusters. Source detection was not performed in regions with an exposure of less than $10 \%$ of the total for the observation. The numbers of total detected X-ray sources are 350 and 365 in Abell 85 and Abell 754, respectively, with only a few sources multiply detected where the FOVs overlap. Our source detection threshold corresponds to $\lesssim 4$ falsely detected X-ray sources (due to a statistical fluctuation) for each observation.

There are two potential ways an X-ray source could be incorrectly associated with an optical source: First, an associated X-ray detection could be a false detection. Second, the positions from an X-ray-detected source and an optical counterpart could randomly overlap. The magnitude of both effects depends on the number of 


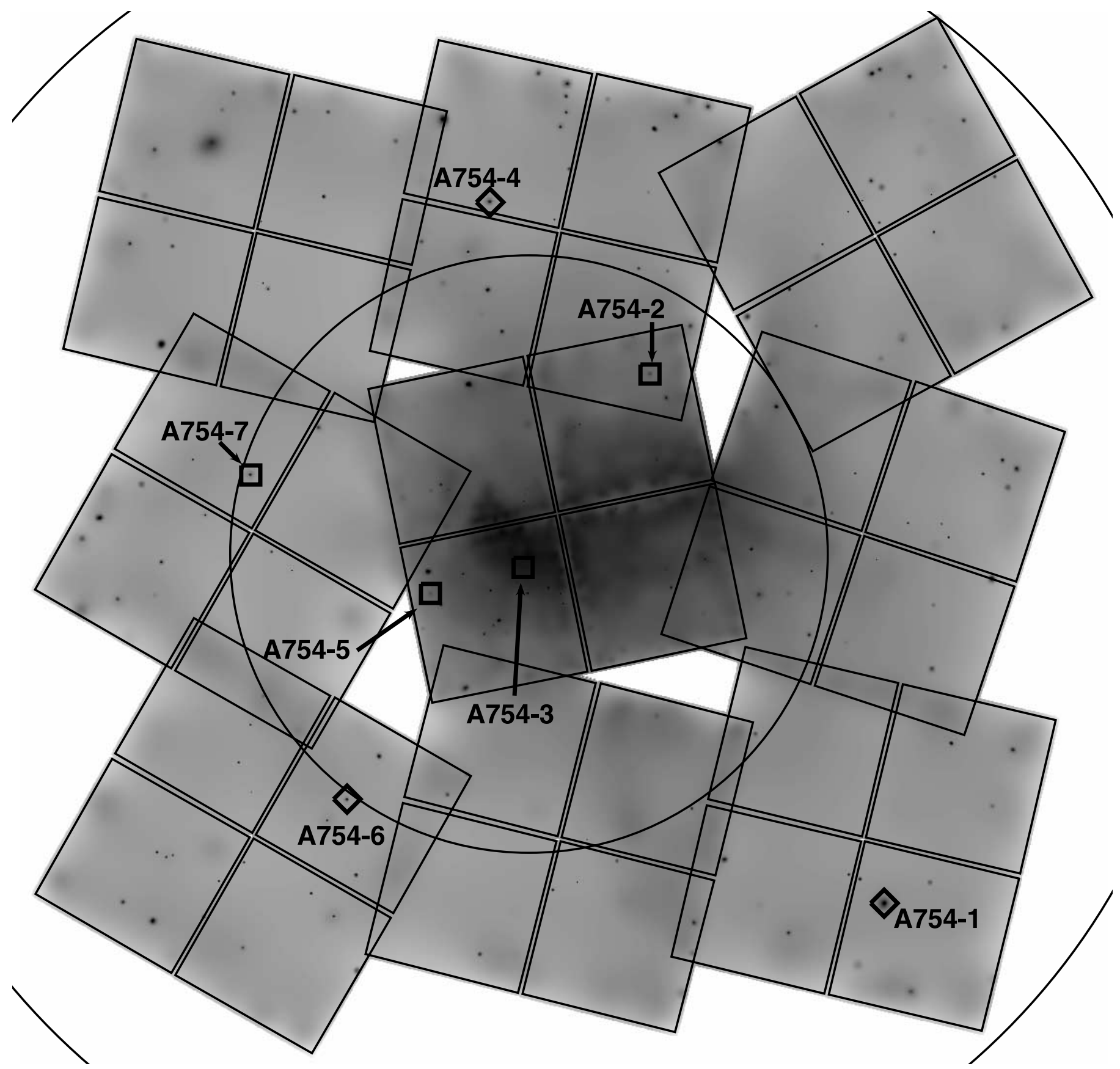

FIG. 2.-Adaptively smoothed Chandra mosaic of Abell 754. Overlays follow the same conventions as Fig. 1.

optical sources and the matching radius used to associate X-ray and optical sources. There are 172,21, and 270 optical members of Abell 85, Abell 89B, and Abell 754, respectively, from CZ03 in the Chandra FOVs, and 50, 4, and 10 additional members from other sources. We first considered a very generous $5^{\prime \prime}$ matching radius for identifying potential X-ray-emitting galaxies. This radius is large due to a $\sim 3^{\prime \prime}$ uncertainty in the position of optical sources from fiber positioning (CZ03) and potential poor localization of the X-ray position due to low-count X-ray data. At this radius, we expect $\lesssim 0.08$ and $\lesssim 0.09$ false associations in the Abell 85 and Abell 754 FOVs, respectively, due to statistical fluctuations above our source detection threshold. By replacing the source detection threshold with the average number of real $\mathrm{X}$-ray sources per pixel, we can calculate the number of false associations due to random overlap. We estimate $\lesssim 0.7$ and $\lesssim 0.9$ false associations in the Abell 85 and Abell 754 FOVs, respectively, from randomly overlapping sources. Since X-ray AGNs must be at the galaxy centers of cluster members, we apply a stricter requirement ( $<2^{\prime \prime}$ offset from the 2MASS galaxy position) in $\S 4$ to classify a source as an X-ray AGN. Thus, we estimate that the expected number of optical galaxies falsely identified as X-ray AGNs is $\lesssim 0.2$ per cluster FOV. In addition, this expected number drops by a factor of 2 if we only consider galaxies with $M_{R}<-20$.

We used the coordinate list generated by wavdetect and the positions of optical galaxies from CZ03 to identify X-ray detections within $5^{\prime \prime}$ of optical counterparts. Due to the sensitivity of the flanking-field observations, we only considered detections and optical galaxies in regions where the local exposure was at least half of the maximum exposure; this eliminates the edges of the ACIS-I chips and the gaps between them. To determine cluster 
TABLE 1

Chandra ACIS-I ObSERvation Log

\begin{tabular}{|c|c|c|c|c|}
\hline $\begin{array}{l}\text { Field } \\
\text { (1) }\end{array}$ & $\begin{array}{c}\text { ObsiD } \\
\text { (2) }\end{array}$ & $\begin{array}{l}\text { Date } \\
\text { (3) }\end{array}$ & $\begin{array}{c}T \\
(\mathrm{ks}) \\
(4)\end{array}$ & $\begin{array}{c}L_{\mathrm{X}, \mathrm{Lim}} \\
\left(10^{40} \mathrm{ergs} \mathrm{s}^{-1}\right) \\
(5)\end{array}$ \\
\hline Abell 85-C .......................... & 0904 (00904) & 2000 Aug 19 & 38.4 & 1.2 \\
\hline Abell 85-SE .......................... & $4881(04881)$ & 2004 Sep 3 & 9.8 & 4.6 \\
\hline 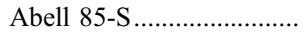 & $4882(04882)$ & 2004 Sep 3 & 9.6 & 4.7 \\
\hline 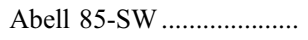 & $4883(04883)$ & 2004 Sep 3 & 9.6 & 4.7 \\
\hline Abell 85-E............................. & $4884(04884)$ & 2004 Sep 3 & 9.6 & 4.7 \\
\hline Abell 85-W ........................ & $4885(04885)$ & 2004 Sep 3 & 9.6 & 4.7 \\
\hline Abell 85-NE........................ & $4886(04886)$ & 2004 Sep 3 & 9.6 & 4.7 \\
\hline Abell 85-N ......................... & 4887 (04887) & 2004 Sep 4 & 10.1 & 4.5 \\
\hline Abell 85-NW ...................... & $4888(04888)$ & 2004 Sep 4 & 9.6 & 4.7 \\
\hline Abell 754-C ........................ & 0507 (00507) & 1999 Oct 30 & 29.8 & 1.3 \\
\hline Abell 754-SE ...................... & $6793(06793)$ & 2006 Jan 18 & 9.9 & 4.6 \\
\hline Abell 754-S........................ & 6794 (06794) & 2006 Jan 27 & 9.9 & 4.6 \\
\hline Abell 754-SW ……................. & $6795(06795)$ & 2006 Jan 28 & 9.6 & 4.7 \\
\hline Abell 754-E........................ & $6796(06796)$ & 2006 Jan 18 & 9.6 & 4.7 \\
\hline Abell 754-W ...................... & 6797 (06797) & 2006 Jan 25 & 10.0 & 4.5 \\
\hline Abell 754-NE........................ & $6798(06798)$ & 2006 Jan 28 & 10.0 & 4.6 \\
\hline Abell 754-N ...................... & $6799(06799)$ & 2006 Jan 28 & 9.6 & 4.7 \\
\hline Abell 754-NW ..................... & $6800(06800)$ & 2006 Feb 15 & 10.2 & 4.5 \\
\hline
\end{tabular}

Notes.-Col. (1): Field targeted. Col. (2): Observation ID of Chandra data. Col. (3): Observation date. Col. (4): Usable exposure. Col. (5): Estimate of the $0.3-8.0 \mathrm{keV}$ luminosity limit of the observation for a $z=0.055$ galaxy.

membership, we adopted the velocity range in Christlein \& Zabludoff (2003) for Abell 85 and Abell 754. For Abell 89B, we determined its cluster properties ourselves (see $\S 3$ ). We found no additional matches when we added additional cluster members from the NASA/IPAC Extragalactic Database (NED). In Table 2 we list the 17 detections that correspond to a galaxy in Abell 85, Abell 89B, or Abell 754. These galaxies are also indicated in Figures 1 and 2. We label the sources in order of right ascension by cluster and list their X-ray position and optical counterpart from CZ03. For each optical counterpart, we adopted the 2MASS position in the Extended Source Catalog (Skrutskie et al. 2006) and recalculated the offset between the X-ray detection and the galaxy center. Using Kim et al. (2007) we have estimated the $\mathrm{X}$-ray positional uncertainty $(1 \sigma)$ due to wavdetect. Our first criteria for an X-ray AGN is that the offset between the X-ray detection and the galaxy is less than $2^{\prime \prime}$, consistent with that used in M06. Since all three detections that fail this criterion have $\sim 1^{\prime \prime}$ positional uncertainty, they are still likely associated with the identified galaxies. We have excluded a detection consistent with the brightest cluster galaxy (BCG) of Abell 85, as this detection also corresponds to the peak in the X-ray flux from the ICM. We also note that a detection corresponding to an Abell 85 member that is likely an X-ray AGN with $L_{\mathrm{X}, B} \sim 1.2 \times 10^{41} \mathrm{ergs} \mathrm{s}^{-1}$ (2MASX J00415019-0925469) was excluded, since it fell in a chip gap of observation 0904 and the photometry is therefore highly uncertain.

For all detections in Table 2, we used ACIS Extract 3.131 to create source extraction regions enclosing $90 \%$ of the flux in the $\mathrm{X}$-ray point-spread function (PSF) and to determine a masking radius that encircled $97 \%$ of the flux. For most of the sources, whose photons had median energies of $\sim 0.6-2.6 \mathrm{keV}$, we determined the regions assuming the PSF at $1.497 \mathrm{keV}$. Since the events for A754-6 had a median energy of $\sim 4.7 \mathrm{keV}$, we used the PSF determined at $4.51 \mathrm{keV}$. For each source, we created background regions just beyond the masking radius with an area 5 times that of the source extraction region. Column (6) of Table 2 indicates the net counts for each source in the $0.3-8.0 \mathrm{keV}$ band, with proper Poisson errors (Gehrels 1986). To estimate the rest-frame 0.3-8.0 keV X-ray luminosity (col. [7]), we folded a power-law spectrum with $\Gamma=1.7$ absorbed by the Galactic column $(3.3 \times$ $10^{20} \mathrm{~cm}^{2}$ for Abell 85 and Abell 89B; $4.4 \times 10^{20} \mathrm{~cm}^{2}$ for Abell 754) through the spectral response at the location of each source. We set the model normalization using $\mathrm{XSPEC}^{7}$ to match the observed net counts, corrected for the mean redshift of the cluster and the enclosed fraction of the flux in the source extraction region.

We note that A754-1 is bright enough that it suffers from events lost to pileup. At $\sim 0.55$ counts per frame, pileup can be relatively minor and require only a small correction or pileup can be more severe and require a larger correction. Since there is no readout streak and the source is shaped like the PSF, the branch with less pileup is more likely correct. Therefore, the luminosity in Table 2 has been corrected assuming that our spectral model has been affected by pileup with a typical grade-migration parameter, $\alpha=$ 0.5 (Davis 2001). We estimate that the correction factor of $\sim 2.4$ is accurate to a factor of $\sim 60 \%$. If the source is more extremely affected by pileup, this will only increase its X-ray luminosity.

By combining the luminosities and counts from Table 2 with our exposure maps, we estimated the limiting X-ray luminosity for each observation. This is listed in Table 1, assuming a redshift of $z=0.055$ near that of Abell 85 and Abell 754. For the more distant Abell 89B, the limiting luminosity is a factor of 2 higher. For consistency with M06, we have calculated this number corresponding to five counts on-axis. However, we caution that this limit is optimistic over an entire ACIS-I FOV for two reasons. First, at $1.5 \mathrm{keV}$ the spatial structure to the quantum efficiency degradation leads to $15 \%$ lower exposure at approximately $10^{\prime}$ off-axis in the latest observations. More importantly, the larger off-axis PSF makes detection of weak sources more difficult. Kim $\&$ Fabbiano (2003) show that at $5^{\prime}$ and $10^{\prime}$ off-axis, $70 \%$ completeness can be expected for 7 and 11 count sources, respectively. We estimate that completeness limits over the entire ACIS-I FOV are about a factor of 4 higher than reported in Table 1 of M06 and

\footnotetext{
${ }^{7}$ See http://heasarc.gsfc.nasa.gov/docs/software/lheasoft.
} 


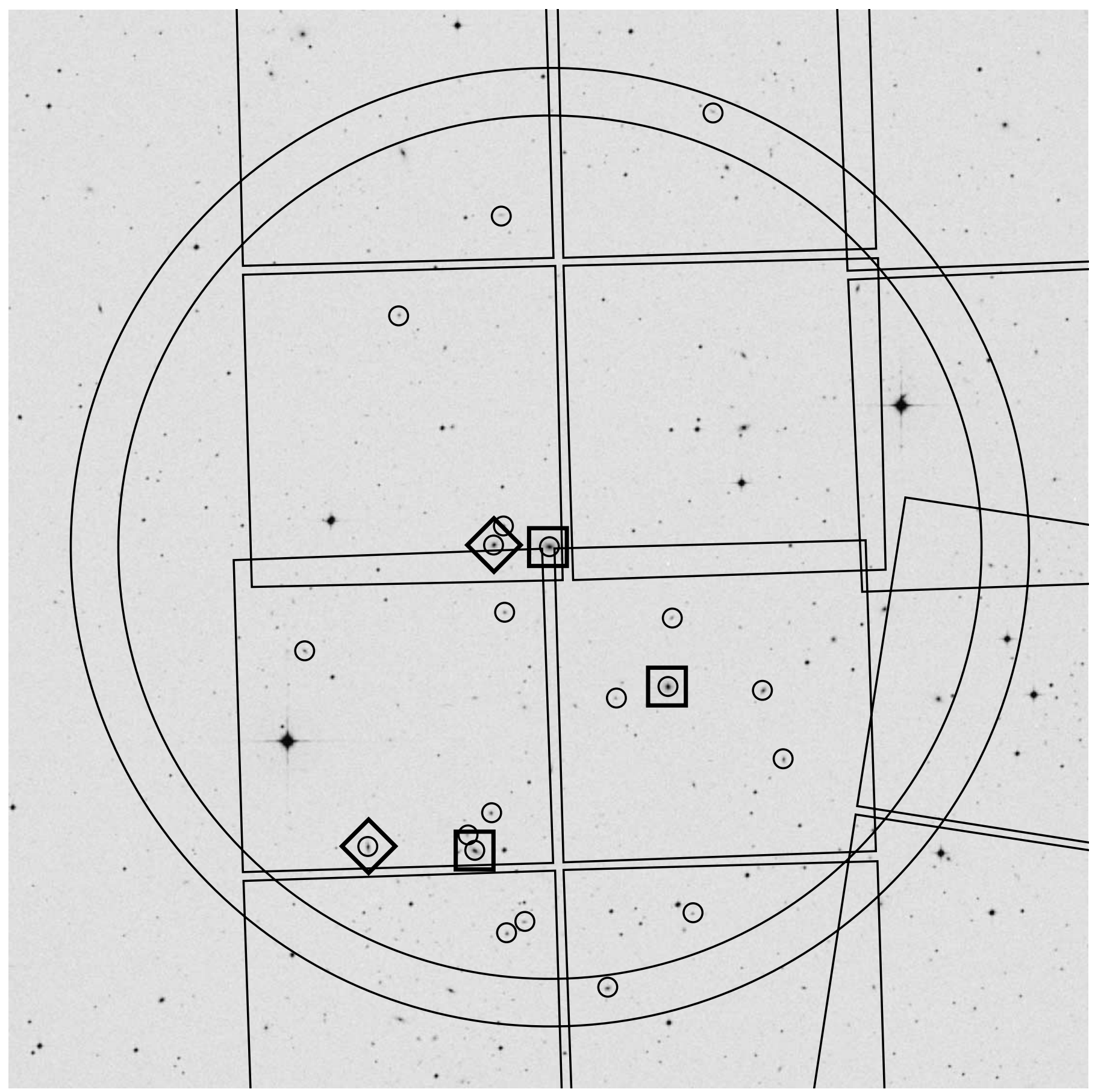

Fig. 3.- Second Palomar Observatory Sky Survey (Red) image centered on the BCG of Abell 89B. Overlays follow the same conventions as Fig. 1, with small circles indicating $M_{R}<-20$ cluster members in the Chandra FOV.

Table 2 of this work. This means that the AGN fraction above $10^{41}$ ergs s$^{-1}$ may be underestimated; however, we estimate that this is a smaller effect than the current error due to the small numbers of AGNs.

\section{3. $z \lesssim 0.08$ CLUSTER SAMPLE}

We required a sample large enough to statistically test which galaxy and cluster properties lead to X-ray AGN activity. This is especially important as not all detected X-ray sources will be X-ray AGNs. To supplement the sample of 17 potential X-ray AGNs in Abell 85, Abell 89B, and Abell 754, we have also included three other $z \lesssim 0.08$ clusters with X-ray-identified AGNs,
Abell 644, Abell 3125, and Abell 3128 (M06; M07). We list the cluster properties in Table 3, adopting the M07 values for the latter three clusters.

In columns (2) and (3) we list the cluster positions. For Abell 85 and Abell 754, we adopted the peak of the ICM as the cluster position. The BCG of Abell 85 is coincident with this peak. In Abell 754, the third brightest galaxy (in $R$ band), A754-3, is embedded in the ICM $\sim 46^{\prime \prime}$ away from this position. This galaxy is located near one of the concentrations of Abell 754 member galaxies.

We list the mean cluster redshift, redshift range of cluster members, and velocity dispersion, with $90 \%$ confidence limit, in 
TABLE 2

X-Ray Properties of Abell 85, Abell 89B, and Abell 754 Galaxies

\begin{tabular}{|c|c|c|c|c|c|c|}
\hline $\begin{array}{l}\text { ID } \\
\text { (1) }\end{array}$ & $\begin{array}{l}\text { CXOU XID } \\
\text { (2) }\end{array}$ & $\begin{array}{c}\text { CZ2003 ID } \\
\text { (3) }\end{array}$ & $\begin{array}{c}\text { 2MASX ID } \\
\text { (4) }\end{array}$ & $\begin{array}{c}\text { Offset } \\
(\operatorname{arcsec}) \\
(5)\end{array}$ & $\begin{array}{l}\text { Net Counts } \\
\text { (6) }\end{array}$ & $\begin{array}{c}L_{\mathrm{X}, B} \\
(7)\end{array}$ \\
\hline A85-1 & J004130.2-091546 & 85A_993[6] & J00413032-0915459 & $1.0(0.3)$ & $160.3_{-148}^{+15.8}$ & $4.2_{-0.4}^{+0.4}$ \\
\hline A $85-2$............................ & J004142.9-092621 & 85A_993[13] & $\mathrm{J} 00414302-0926219$ & $0.8(0.4)$ & $21.0_{-6.1}^{+7.1}$ & $0.5_{-0.4}^{+0.4}$ \\
\hline A $85-3$............................. & J004146.7-092313 & 85A_993[12] & $\mathrm{J} 00414681-0923129$ & $0.8(0.3)$ & $7.6_{-3.0}^{+4.1}$ & $0.2_{-0.1}^{-0.4}$ \\
\hline . & J004244.7-093312 & 85A_993[86] & $\mathrm{J} 00424470-0933162$ & $3.4(1.1)$ & $5.8_{-24}^{+3.6}$ & $0.6_{-0.3}^{+0.1}$ \\
\hline 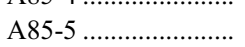 & J004311.5-093816 & 85A_993[47] & $\mathrm{J} 00431162-0938163$ & $0.5(0.4)$ & $30.0_{-5.5}^{+6.5}$ & $5.2_{-0.9}^{+1.3}$ \\
\hline 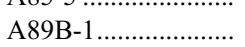 & J004242.0-091731 & 85A_993[80] & J00424193-0917312 & $1.7(1.2)$ & $\begin{array}{r}7.0_{-5.5}^{+4.0} \\
7.0_{-2.8}^{+4}\end{array}$ & $\begin{array}{l}.2-0.9 \\
1.5_{-0.6}^{+0.9}\end{array}$ \\
\hline A89B-2 & J004254.8-091349 & 85A_993[81] & J00425466-0913493 & $2.3(1.4)$ & $\begin{array}{r}13.0_{-2.8}^{+4.9} \\
13.0_{-38}^{+4.9}\end{array}$ & $3.2_{-0.9}^{+1.6}$ \\
\hline A89B-3 & J004300.6-091346 & 85A_993[57] & J00430067-0913463 & $0.9(0.6)$ & $\begin{array}{l}1.0_{-3.8} \\
64.0_{-81}^{+9.1}\end{array}$ & $\begin{array}{r}5.2_{-0.9}^{-0.9} \\
14.3_{-1.8}^{+2.0}\end{array}$ \\
\hline 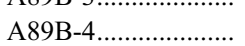 & J004302.7-092151 & 85A_993[59] & J00430270-0921513 & $0.5(1.3)$ & $\begin{array}{r}3.0_{-8.1} \\
3.0_{-17}^{+2.9}\end{array}$ & $\begin{array}{r}4 . J_{-1.8} \\
0.6_{-0.6}^{+0.6}\end{array}$ \\
\hline A89B-5....................... & $\mathrm{J} 004314.0-092144$ & 85A_993[60] & J00431418-0921453 & $1.6(0.6)$ & $8.6_{-3.0}^{+4.1}$ & $1.8_{-0.6}^{+0.3}$ \\
\hline 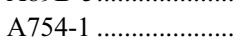 & J090802.1-095937 & 754A_494[25] & J09080217-0959378 & $0.5(0.1)$ & $1697.0_{-413}^{+42.3}$ & $389.7^{-0.633 .8^{\mathrm{a}}}$ \\
\hline A754-2 …...................... & J090852.2-093149 & 754A_494[100] & J09085229-0931507 & $1.9(0.9)$ & $38.8_{-7.5}^{+8.5}$ & $1.2_{-0.2}^{+0.3 .8}$ \\
\hline A754-3 ...................... & J090919.2-094159 & 754A_494[9] & J09091923-0941591 & $0.2(0.4)$ & $13.5_{-4.8}^{+5.8}$ & $0.4_{-0.1}^{+0.2}$ \\
\hline A754-4 ……................ & J090926.3-092247 & 754A_494[93] & J09092633-0922471 & $0.6(0.3)$ & $40.4_{-64}^{+7.5}$ & $4.0_{-06}^{+0.7}$ \\
\hline 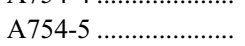 & J090939.0-094321 & 754A_494[106] & J09093913-0943233 & $3.0(1.1)$ & $21.2_{-6.6}^{+7.6}$ & $0.6_{-0.2}^{+0.6}$ \\
\hline 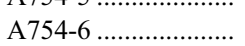 & J090956.8-095409 & 754A_393[55] & J09095685-0954093 & $0.8(0.4)$ & $32.4_{-5.7}^{+6.8}$ & $3.5_{-0.0}^{+0.2}$ \\
\hline A754-7 ……............ & J091017.3-093707 & 754A_494[76] & J09101737-0937068 & $1.2(0.4)$ & $14.6_{-3.9}^{+5.0}$ & $1.4_{-0.4}^{+0.5}$ \\
\hline
\end{tabular}

Notes._Col. (1): ID used in this paper. Col. (2): X-ray object ID. Col. (3): ID from CZ03. Col. (4): 2MASS Extended Source Catalog ID of counterpart. Col. (5): Offset between X-ray and near-IR position with the an estimate of the $1 \sigma$ statistical uncertainty of the X-ray position in the parentheses. Col. (6): Net X-ray counts detected in observed frame $0.3-8.0 \mathrm{keV}$ band with exact Gehrels errors (Gehrels 1986). Col. (7): X-ray luminosity in rest-frame $0.3-8.0 \mathrm{keV}$ band in units of $10^{41} \mathrm{ergs} \mathrm{s}^{-1}$. The X-ray luminosity was calculated assuming a $\Gamma=1.7$ power law with corrections for Galactic absorption and the enclosed fraction of the PSF used to extract the counts.

${ }^{\text {a }} \mathrm{X}$-ray analysis affected by pile-up. The luminosity correction factor of $\sim 2.4$ is uncertain to $\sim 60 \%$.

columns (4)-(6). We adopted the values of CZ03 for Abell 85 and Abell 754; however, we increased their $1 \sigma$ uncertainties in velocity dispersion by a factor of 1.6 to match our confidence limits. For Abell 89B, the Christlein \& Zabludoff (2003) data suggested that its members were in the $0.06<z<0.09$ range. We calculated membership via the biweight estimator for center and scale, following M07, adding additional nearby galaxies with velocity data in the NED to the CZ03 sample. We iteratively determined 29 galaxies were within $5 \sigma$ of the cluster mean velocity and the $r_{200}$, assuming the BCG was the center of the cluster. Of the 29 galaxies, our FOV overlapped with 25. We used the jackknife of the biweight estimator to determine the $90 \%$ confidence limit for the velocity dispersion. The symmetric confidence limit (eq. [22] of Beers et al. 1990) was chosen for consistency with M06 and M07.

To characterize the extent of the clusters and best compare the spatial distributions of cluster AGNs, we determined the $r_{200}$ of each cluster following equation (A1) of Treu et al. (2003). These are listed in column (7).

\section{GALAXY PROPERTIES OF X-RAY SOURCES}

\subsection{X-Ray AGN Identification}

Near the luminosity limits of these observations, there are three potential sources of X-ray emission: X-ray binaries, a hot ISM, and a central AGN (e.g., Sivakoff et al. 2003, 2004). X-ray binaries with low-mass companions (LMXBs) are sensitive to the total stellar mass of a galaxy, while X-ray binaries with highmass companions (HMXBs) are sensitive to recent star formation (Grimm et al. 2003; Kim \& Fabbiano 2004). From a sample of 14 nearby galaxies, Kim \& Fabbiano (2004) derived a (linear) relation between the total X-ray luminosity of LMXBs within the galaxy and the $B$ - or $K_{s}$-band luminosity. We prefer the latter relation, as $K_{s}$ band is a better tracer of stellar mass, and the relation has a smaller dispersion:

$$
L_{\mathrm{X}, B}=(2.0 \pm 0.8) \times 10^{29} \mathrm{ergs} \mathrm{s}^{-1} / L_{K_{s}, K 20, \odot},
$$

TABLE 3

Cluster Properties

\begin{tabular}{|c|c|c|c|c|c|c|c|}
\hline $\begin{array}{l}\text { Cluster } \\
\text { (1) }\end{array}$ & $\begin{array}{l}\alpha_{c} \\
(2)\end{array}$ & $\begin{array}{l}\delta_{c} \\
(3)\end{array}$ & $\begin{array}{c}z \\
(4)\end{array}$ & $\begin{array}{c}z_{1}, z_{2} \\
\text { (5) }\end{array}$ & $\begin{array}{c}\sigma \\
(6)\end{array}$ & $\begin{array}{l}r_{200} \\
(7)\end{array}$ & $\begin{array}{c}\text { Reference } \\
\text { (8) }\end{array}$ \\
\hline Abell 85 & 004150.4 & -091811 & 0.0554 & $0.0448,0.0658$ & $993(85)$ & 2.4 & 1 \\
\hline Abell 89B.................... & 004254.6 & -091350 & 0.077 & $0.0692,0.0850$ & $474(155)$ & 1.1 & 2 \\
\hline 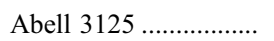 & 032517.9 & -532937 & 0.0616 & $0.0530,0.0700$ & $475(94)$ & 1.1 & 3 \\
\hline Abell 3128 & 033043.8 & -523130 & 0.0595 & $0.0435,0.0755$ & $906(74)$ & 2.1 & 3 \\
\hline Abell 754 & 090918.0 & -094117 & 0.0546 & $0.0446,0.0632$ & $953(64)$ & 2.3 & 1 \\
\hline Abell 644 .......................... & 081725.6 & -073045 & 0.0701 & $0.0531,0.0871$ & $952(382)$ & 2.2 & 3 \\
\hline
\end{tabular}

Notes.-Sample of $z \lesssim 0.08$ clusters with X-ray-identified AGNs. Col. (1): Cluster name. Columns (2) and (3): Right ascension and declination of the cluster center for epoch J2000. Units of right ascension are hours, minutes, and seconds, and units of declination are degrees, arcminutes, and arcseconds. Col. (4): Redshift. Col. (5): Redshift range of cluster members. Col. (6): Velocity dispersion and uncertainty (90\%). Col. (7): $r_{200}$ in Mpc. Col. (8): Reference for velocity information.

REFERENCES.-(1) CZ03; (2) this paper; (3) M07. 
where $L_{K_{s}, K 20, \odot}$ is the $K_{s}$-band luminosity within the $K_{s}=$ $20 \mathrm{mag} \operatorname{arcsec}^{2}$ isophote, assuming $M_{\odot, K_{s}}=3.33$. We caution that most of these galaxies in this archival sample were originally targeted due to their X-ray properties. These galaxies are roughly divided into X-ray-bright galaxies, galaxies with significantly higher X-ray to optical flux ratios that are dominated by the diffuse gas, and X-ray-faint galaxies, galaxies with lower $\mathrm{X}$-ray to optical flux ratios that are dominated by the X-ray binaries. The X-ray-bright galaxies should be relatively free from bias on the total LMXB X-ray luminosities in these systems. As studying LMXBs was often the primary science driver for targeting the X-ray-faint galaxies, these galaxies were often selected based on their X-ray luminosities or X-ray to optical flux ratios. Due to such selection criteria, X-ray-faint galaxies whose X-ray luminosities are toward the lower end of the intrinsic relation between X-ray luminosity from LMXBs and stellar mass are less likely to be targeted by observers. Thus, the above relation may overestimate the intrinsic relation. To estimate the X-ray luminosity from HMXBs, the SFR is needed. Assuming a $\Gamma=1.7 \mathrm{X}$-ray spectrum, we can convert the relation found in Grimm et al. (2003) to a $L_{\mathrm{X}, B}$ value, such that

$$
L_{\mathrm{X}, B}=1.0 \times 10^{40} \frac{\mathrm{SFR}}{M_{\odot} \mathrm{yr}^{-1}} \operatorname{ergs~\mathrm {s}^{-1}} .
$$

Since the ISM is thought to have a stellar origin, a rough correspondence with stellar mass is expected; however, at a given stellar mass there is a wide range of ISM luminosities and the relation to stellar mass is known to be nonlinear. We adopt the Sun et al. (2007) relation:

$$
\log L_{\mathrm{X}, S}=39.40+(1.63 \pm 0.13) \log \left(\frac{L_{K_{s}, \mathrm{ttl}, \odot}}{10^{11}}\right)
$$

where the soft $(0.5-2.0 \mathrm{keV})$ band X-ray luminosity, $L_{\mathrm{X}, S}$ is calculated assuming an ISM spectral model and $L_{K_{s}, t \mathrm{tl}, \odot}$ is the total $K_{s}$-band luminosity. This relation is derived including the effects of upper limits for nondetections of the ISM. For the galaxies in our cluster sample, we have estimated that $L_{K_{s}, \text { ttl }} \sim 1.23 L_{K_{s}, K 20}$ and that the $L_{\mathrm{X}, B}$ for a $\Gamma=1.7$ power law is $\sim 1.9$ times the $L_{\mathrm{X}, S}$ for $k T=0.7 \mathrm{keV}$ gas with 0.8 solar abundance when requiring that the observed $0.3-8.0 \mathrm{keV}$ count rates match. Note that applying just a luminosity cut of $L_{\mathrm{X}, B}=10^{41} \mathrm{ergs} \mathrm{s}^{-1}$ to identify AGNs can be contaminated by galaxies without AGNs if either $L_{K_{s}, \odot} \gtrsim 2.5 \times 10^{11}$ or SFR $\gtrsim 10 M_{\odot} \mathrm{yr}^{-1}$.

In Table 4 we list the optical/near-IR magnitudes for galaxies in our sample of clusters. In column (2) we list the observed $R$-band magnitude. We list the references for these magnitudes in column (5). The absolute $R$-band magnitude, including extinction corrections $\left[A_{R}=2.64 E(B-V)\right.$; Schlegel et al. 1998], are listed in column (3), assuming a distance corresponding to the mean redshift of each cluster. As in Martini et al. (2006), we applied corrections for bandpass shifting and stellar evolution based on a simple stellar population model with solar metallicity and formation redshift of $z=3$ (Bruzual \& Charlot 2003). At these redshifts the corrections to the $R$-band magnitudes are small (0.06-0.08). All X-ray sources are in galaxies with $M_{R}<-20$. For comparison, we note that the knee of local galaxy luminosity functions occurs at $M_{R}^{*}=-21.15$ (CZ03). In column (4) we list the absolute $K_{S}$-band magnitude with extinction corrections $\left[A_{K_{s}}=\right.$ $0.28 E(B-V)$; Majewski et al. 2003], where we have used the 2MASS magnitude within the $K_{s}=20 \mathrm{mag} \operatorname{arcsec}^{2}$ isophote (Skrutskie et al. 2006). The correction for bandpass shifting and
TABLE 4

\begin{tabular}{|c|c|c|c|c|}
\hline $\begin{array}{l}\text { ID } \\
(1)\end{array}$ & $\begin{array}{l}m_{R} \\
(2)\end{array}$ & $\begin{array}{l}M_{R} \\
(3)\end{array}$ & $\begin{array}{c}M_{K_{s}, K 20} \\
\quad(4)\end{array}$ & $\begin{array}{l}\text { Ref. } \\
(5)\end{array}$ \\
\hline A $85-1^{\mathrm{a}} \ldots \ldots$ & 14.32 & -22.81 & -25.14 & 1 \\
\hline 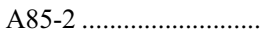 & 14.43 & -22.70 & -25.43 & 1 \\
\hline A85-3 ........................... & 15.86 & -21.27 & -24.05 & 1 \\
\hline 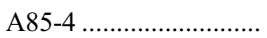 & 14.81 & -22.10 & -24.98 & 1 \\
\hline 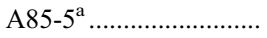 & 15.03 & -22.22 & -24.75 & 1 \\
\hline A89B-1 ........................... & 14.18 & -23.08 & -25.81 & 1 \\
\hline 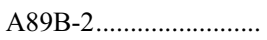 & 15.03 & -23.71 & -26.37 & 1 \\
\hline A $89 B-3^{a}$. & 15.17 & -22.85 & -25.51 & 1 \\
\hline 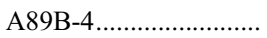 & 14.92 & -22.72 & -25.81 & 1 \\
\hline A89B-5 ${ }^{\mathrm{a}} \ldots \ldots \ldots \ldots \ldots \ldots$ & 14.09 & -22.03 & -25.19 & 1 \\
\hline 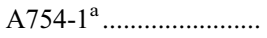 & 14.40 & -22.79 & -25.69 & 1 \\
\hline A754-2 ........................ & 14.60 & -22.54 & -25.30 & 1 \\
\hline 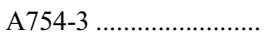 & 14.05 & -23.13 & -25.63 & 1 \\
\hline A754-4 & 14.24 & -22.90 & -25.79 & 1 \\
\hline 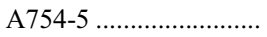 & 14.28 & -22.87 & -25.60 & 1 \\
\hline A754-6 ${ }^{\mathrm{a}} \ldots \ldots \ldots \ldots \ldots \ldots \ldots$ & 15.84 & -21.33 & -24.10 & 1 \\
\hline A754-7 ......................... & 13.93 & -23.19 & -26.00 & 1 \\
\hline A3125-1 ${ }^{\mathrm{a}} \ldots \ldots \ldots \ldots \ldots \ldots$ & 15.77 & -21.56 & -24.66 & 2 \\
\hline A3125-2 ...................... & 14.93 & -22.39 & -25.62 & 2 \\
\hline A3125-3 ...................... & 15.24 & -22.08 & -25.43 & 2 \\
\hline A3125-4 ........................ & 15.16 & -22.17 & -25.42 & 2 \\
\hline A $3125-5^{a} \ldots \ldots$. & 15.97 & -21.36 & -24.54 & 2 \\
\hline A3125-6 ${ }^{\mathrm{a}} \ldots \ldots \ldots \ldots \ldots \ldots$ & 14.97 & -22.36 & -24.18 & 3 \\
\hline A3128-1 ......................... & 15.24 & -22.01 & $\ldots$ & 2 \\
\hline A3128-2 $2^{a} \ldots \ldots \ldots \ldots \ldots \ldots$ & 17.17 & -20.08 & $\ldots$ & 2 \\
\hline A3128-3 ..................... & 16.21 & -21.04 & -24.16 & 4 \\
\hline A3128-4 $4^{\mathrm{a}} \ldots \ldots \ldots \ldots \ldots \ldots$ & 14.81 & -22.43 & -26.09 & 2 \\
\hline A3128-5 ...................... & 15.65 & -21.60 & -24.62 & 5 \\
\hline A3128-6 $6^{\mathrm{a}} \ldots \ldots \ldots \ldots \ldots \ldots$ & 16.82 & -20.43 & -23.22 & 2 \\
\hline A3128-7 ....................... & 15.01 & -22.23 & -25.77 & 2 \\
\hline A3128-8 ........................ & 15.28 & -21.97 & -26.02 & 2 \\
\hline A3128-9 $9^{a} \ldots \ldots \ldots \ldots \ldots$ & 16.41 & -20.83 & -23.76 & 2 \\
\hline A3128-10 ...................... & 14.60 & -22.65 & -26.33 & 2 \\
\hline A644-1 ${ }^{\mathrm{a}} \ldots \ldots \ldots \ldots \ldots \ldots$ & 16.63 & -21.94 & -24.80 & 2 \\
\hline A644-2 ${ }^{\mathrm{a}} \ldots \ldots \ldots \ldots \ldots \ldots \ldots$ & 15.90 & -21.23 & -24.22 & 2 \\
\hline
\end{tabular}

Optical/Near-IR Magnitudes of X-Ray Galaxies

Notes.-Optical/near-IR measurements of X-ray identified galaxies in six $z<0.08$ clusters. Col. (1): ID from this paper or M06. Col. (2): Observed $R$-band magnitude. Col. (3): Extinction-corrected rest-frame absolute $R$-band magnitude. Col. (4): Extinction-corrected rest-frame absolute $K_{s}$-band magnitude within the $K_{s}=20 \mathrm{mag} \operatorname{arcsec}^{2}$ isophote. Col. (5): Reference for $R$-band magnitude.

a Galaxy selected as X-ray AGN.

REFERENCES.-(1) CZ03; (2) M06; (3) Lauberts \& Valentijn 1989; (4) Caldwell \& Rose 1997; (5) Katgert et al. 1998.

stellar evolution to the $K$-band magnitudes are larger (0.24-0.32) than those applied to the $R$-band magnitudes. While we do not have robustly measured SFR for these galaxies, we place rough limits on the SFR in $\S 4.3$.

In Figure 4 we plot the X-ray versus $K_{s}$-band luminosity for galaxies in our cluster sample. The errors for the X-ray luminosity are calculated from the errors in the count rates alone, except for A754-1, for which the errors arise from uncertainty in the pileup correction. To estimate the near-IR luminosity for the two galaxies that were not in the 2MASS Extended Source Catalog, we used the relation between the standard aperture $K_{s}$ magnitude in the Point Source Catalog and the $K_{s}$-band isophotal magnitude for the other galaxies. These two galaxies are indicated with their larger dashed error bars. Galaxies with X-ray luminosities newly measured for this paper are indicated with filled symbols in Figure 4. We overlay the $1 \sigma$ ranges of the Kim \& Fabbiano (2004) and Sun et al. (2007) relations after correcting the latter to isophotal optical luminosities and $L_{\mathrm{X}, B}$ assuming a $\Gamma=1.7$ power 


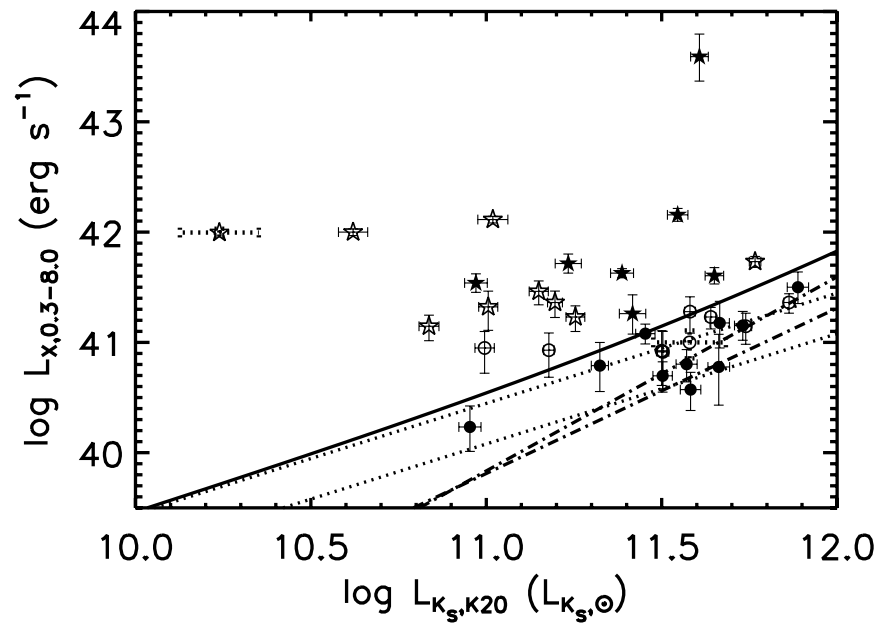

FIG. 4.-Broadband X-ray luminosity, $L_{\mathrm{X}, B}$, vs. the near-IR luminosity enclosed in the $K_{s}=20 \mathrm{mag} \operatorname{arcsec}^{2}$ isophote, $L_{K_{s}, K 20}$, for X-ray-detected galaxies in the cluster sample from Table 3. The $1 \sigma$ range of X-ray emission expected from LMXBs (dotted line; Kim \& Fabbiano 2004) and diffuse gas (dashdotted line; Sun et al. 2007) are displayed. Galaxies that have $L_{\mathrm{X}, B}$ brighter than $10^{41} \mathrm{ergs} \mathrm{s}^{-1}$ and more than $1 \sigma$ away from the sum of the upper limits for LMXBs and diffuse gas (solid line) are considered X-ray AGNs and are marked by stars. Filled and open symbols indicate galaxies from this paper and Martini et al. (2006), respectively. Two of the galaxies from M06 had no 2MASS Extended Source Catalog counterpart and have estimated $L_{K_{S}{ }_{20}}$ and larger errors (thick dotted bars). The most luminous X-ray source, A754-1, has been corrected for pileup, which is uncertain to $\sim 60 \%$.

law. The solid line indicates the sum of the upper limits from both relations.

We classify a galaxy as an X-ray AGN if the following conditions are met: $L_{\mathrm{X}, B}>10^{41} \mathrm{ergs} \mathrm{s}^{-1}, L_{\mathrm{X}, B}$ more than $1 \sigma$ higher than the sum of the $1 \sigma$ upper limits to the Kim \& Fabbiano (2004) and Sun et al. (2007) relations, and an optical counterpart within $2^{\prime \prime}$. These galaxies are indicated by a note in Table 4 and with a star in Figure 4. One source is marginally above the sum of the $1.3 \sigma$ upper limits to the Kim \& Fabbiano (2004) and Sun et al. (2007) relations, A89B-5; all other sources are above the sum of the $2.7 \sigma$ upper limits of the relations. Since our X-ray luminosity is derived for a point source and not the entire galaxy, we note that the total galaxy X-ray luminosity will be even larger than that in Figure 4 if there is a contribution from the extended emission of the distribution of LMXBs or ISM. Thus, the only likely contaminating sources in this sample of X-ray AGNs are galaxies with SFR $\gtrsim$ $10 M_{\odot} \mathrm{yr}^{-1}$. We argue below that such contamination does not seem likely for our sample. We also note that A3128-3 would be classified as an X-ray AGN if we do not impose an X-ray luminosity cut, i.e., it has a close optical counterpart, is above the sum of the LMXB and ISM relations, but has $L_{\mathrm{X}, B}<10^{41} \mathrm{ergs} \mathrm{s}^{-1}$.

Although one could consider adding additional requirements to classify a source as an X-ray AGN based on its X-ray data, in particular, estimates of its spectrum (e.g., through hardness ratios or quantiles) and spatial decomposition into a point source and extended galactic emission, the quality of the data for the lower luminosity sources is insufficient. First, only $20 \%$ of the X-raydetected galaxies have more than 50 counts. There would be little to no discriminating power for the vast majority of our sample. Second, it is unclear that a spectral selection using hardness ratios or quantiles is appropriate. M06 found that the spectroscopically identified AGNs were those least consistent with unobscured, $\Gamma=1.7$, power-law emission. While one might hope to discriminate the soft emission of diffuse gas from harder power-law emission, some AGNs have ultrasoft spectra, which corresponds to steep
TABLE 5

Optical Spectral Properties of X-Ray Galaxies

\begin{tabular}{|c|c|c|c|}
\hline $\begin{array}{l}\text { ID } \\
\text { (1) }\end{array}$ & $\begin{array}{c}\mathrm{EW}[\mathrm{O} \text { II] } \\
(2)\end{array}$ & $\begin{array}{c}\mathrm{EW}[\mathrm{O} \text { III] } \\
(3)\end{array}$ & $\begin{array}{c}\mathrm{EW} \mathrm{H} \beta \\
(4)\end{array}$ \\
\hline A85-1 ...................... & $37.88 \pm 2.31$ & $84.88 \pm 3.20$ & $4.61 \pm 5.04$ \\
\hline A85-2 ...................... & $-0.21 \pm 0.69$ & $0.15 \pm 0.32$ & $-0.41 \pm 0.18$ \\
\hline A85-3 ….................. & $1.53 \pm 1.11$ & $0.02 \pm 0.51$ & $-0.29 \pm 0.31$ \\
\hline 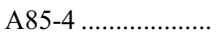 & $4.06 \pm 2.53$ & $0.21 \pm 0.25$ & $-0.08 \pm 0.28$ \\
\hline A85-5 ...................... & $1.08 \pm 1.04$ & $-0.36 \pm 0.48$ & $-0.34 \pm 0.27$ \\
\hline A89B-1 ................... & $-0.49 \pm 0.59$ & $0.14 \pm 0.51$ & $-0.03 \pm 0.22$ \\
\hline A89B-2.. & $3.30 \pm 1.90$ & $-0.29 \pm 0.28$ & $0.00 \pm 0.25$ \\
\hline A89B-3.. & $1.29 \pm 1.03$ & $-0.15 \pm 0.24$ & $-0.59 \pm 0.19$ \\
\hline A89B-4... & $4.29 \pm 1.79$ & $1.40 \pm 0.66$ & $-0.09 \pm 0.26$ \\
\hline A89B-5 .................. & $21.99 \pm 2.45$ & $25.47 \pm 1.65$ & $-0.11 \pm 0.63$ \\
\hline A754-1 .................... & $3.41 \pm 2.06$ & $-0.18 \pm 0.30$ & $-0.25 \pm 0.26$ \\
\hline A754-2 ................... & $4.24 \pm 2.38$ & $-0.23 \pm 0.30$ & $-0.34 \pm 0.22$ \\
\hline A754-3 ................. & $-0.30 \pm 0.64$ & $-0.10 \pm 0.16$ & $-0.39 \pm 0.13$ \\
\hline A754-4 .................... & $1.51 \pm 1.20$ & $0.36 \pm 0.46$ & $-0.22 \pm 0.19$ \\
\hline A754-5 .................. & $-0.42 \pm 0.60$ & $0.28 \pm 0.32$ & $-0.10 \pm 0.15$ \\
\hline A754-6 .................. & $10.36 \pm 1.42$ & $11.05 \pm 1.37$ & $0.20 \pm 0.55$ \\
\hline
\end{tabular}

Notes.-Optical spectral properties of X-ray identified galaxies in Abell 85 Abell 89B, and Abell 754. Col. (1): ID from this paper. Col. (2): Equivalent width of [O II] emission. Col. (3): Equivalent width of [O III] emission. Col. (4): Equivalent width of $\mathrm{H} \beta$ emission without correction for absorption. Abell 754-7 is not included due to its spectrum having low $\mathrm{S} / \mathrm{N}$.

power-law photon indices, $\Gamma \gtrsim 3$ (Puchnarewicz et al. 1992). This highlights the need for deep-enough observations for which spectral modeling can be done to detect the iron L-shell hump characteristic of diffuse gas (e.g., Sun et al. 2007). Since most of our X-ray-detected galaxies have less than 50 counts, spatial decomposition of the X-ray emission would also not be useful for the vast majority of galaxies in our sample.

Based on Kim \& Fabbiano (2003) we estimate that the completeness limits over the entire ACIS-I FOV is approximately 4 times the X-ray luminosity in Table 1 of M06 and Table 2 of this work. This suggests that the central observation of Abell 85 and Abell 754 are incomplete at $L_{\mathrm{X}, B} \lesssim 5 \times 10^{40} \mathrm{ergs} \mathrm{s}^{-1}$, while the flanking-field observations are incomplete for $L_{\mathrm{X}, B} \lesssim 2 \times$ $10^{41} \mathrm{ergs} \mathrm{s}^{-1}$. Since Abell 89B is in flanking-field observations of Abell 85 and is more distant, it is incomplete for $L_{\mathrm{X}, B} \lesssim 4 \times$ $10^{41} \mathrm{ergs} \mathrm{s}^{-1}$. Abell 644 and Abell 3128 are incomplete for $L_{\mathrm{X}, B} \lesssim 10^{41} \mathrm{ergs} \mathrm{s}^{-1}$, while Abell 3125 is incomplete for $L_{X, B} \lesssim$ $2 \times 10^{41} \mathrm{ergs} \mathrm{s}^{-1}$. Although there is a gap between $10^{41} \mathrm{ergs} \mathrm{s}^{-1}$ and the completeness limits in some areas of the clusters, we estimate that the completeness in this gap is above $50 \%$. Since only one X-ray AGN, A89B-5, is detected in the gap between $L_{\mathrm{X}, B}>$ $10^{41} \mathrm{ergs} \mathrm{s}^{-1}$ and its completeness limit, we estimate that we are not likely to be missing more than one or two X-ray AGNs due to incompleteness.

\subsection{Spectroscopically Identified AGNs in Abell 85, Abell 89B, and Abell 754}

In addition to measuring the redshifts of galaxies in Abell 85, Abell 89B, and Abell 754, the spectroscopy described in CZ03 and Christlein \& Zabludoff (2005) yielded measurements of the equivalent widths of the $[\mathrm{O}$ II $] \lambda 3727$ doublet, $\left[\mathrm{O}_{\mathrm{III}}\right] \lambda 5007$, and $\mathrm{H} \beta \lambda 4861$ emission lines. The last is not corrected for any $\mathrm{H} \beta$ absorption. These emission lines can be indicative of ionization from an AGN and/or current, unobscured star formation. These values are listed in Table 5 for X-ray-detected galaxies. We used our spectroscopic measurements to check for AGNs identifiable by their optical spectra among our X-ray-detected galaxies in 
Abell 85, Abell 89B, and Abell 754. The spectroscopic identification of AGNs in our other clusters was previously discussed in M06.

Only three of the X-ray-detected galaxies, A85-1, A89B-5, and A754-6, show emission lines detected at $>3 \sigma$. All three galaxies, which are classified as X-ray AGNs, have significant detections of [O $\mathrm{II}]$ and $\left[\mathrm{O}_{\mathrm{III}}\right]$; however, none of them have $\mathrm{H} \beta$ emission. To conservatively correct for potential absorption, we have added the emission-corrected $\mathrm{H} \beta$ absorption equivalent widths of $5 \AA$ found for poststarburst galaxies in the Sloan Digital Sky Survey (C. Tremonti, private communication) to all measurements of $\mathrm{H} \beta$. Both A85-1 and A89B-5 are spectroscopically classified as $\mathrm{AGNs}$ via the $[\mathrm{O}$ III $] / \mathrm{H} \beta$ versus $\left[\mathrm{O}_{\mathrm{II}}\right] / \mathrm{H} \beta$ diagnostic (Rola et al. 1997; Lamareille et al. 2004):

$$
\log \left(\frac{[\mathrm{O} \text { III }]}{\mathrm{H} \beta}\right)>\frac{0.14}{\left[\mathrm{O}_{\mathrm{II}}\right] / \mathrm{H} \beta-1.45}+0.83
$$

No other galaxies in the Chandra FOVs of these clusters are spectroscopically identified as AGNs with our emission-line data. Since A85-1 has been previously identified as a Seyfert galaxy (Durret et al. 2005) and A89B-5 has been classified as a QSO by SDSS (Adelman-McCarthy et al. 2007), their identifications as spectroscopically identified AGN appear secure.

In addition to the galaxies we identify as spectroscopic AGNs, two other X-ray-detected galaxies have been previously identified as AGNs based on their optical spectral properties. A85-2 was identified as a Seyfert 2 (Hewitt \& Burbidge 1991); however, we note that the redshift associated with this identification (0.0453) does not match our measured redshift of the galaxy (0.0564). A85-3 was identified as an AGN based on the limit to its $[\mathrm{N}$ II $] / \mathrm{H} \alpha$ ratio (Hornschemeier et al. 2005). Neither A85-2 nor A85-3 was identified as an X-ray AGN, because their low $\mathrm{X}$-ray luminosities $\left(L_{\mathrm{X}, B}<10^{41} \mathrm{ergs} \mathrm{s}^{-1}\right)$ were consistent with emission from their LMXB populations. These sources illustrate that although current X-ray observations allow identification of low-luminosity AGNs, some lower luminosity AGNs are still being missed. Another famous example is that the X-ray emission from the core and jet of M87 (e.g., Marshall et al. 2002) would not be luminous enough to be classified as an X-ray AGN with our criteria.

\subsection{Star Formation Rates}

Since HMXBs associated with star formation may also lead to X-ray emission, it is important to evaluate whether an X-raydetected galaxy has a high SFR. We use optical spectroscopy to constrain the current SFR through the [O II] equivalent width. Rough limits on the SFR for X-ray galaxies can be estimated from detections and limits based on the Infrared Astronomy Satellite (IRAS) Faint Source Catalog (Moshir et al. 1990). For Abell 85, Abell 89B, Abell 754, and Abell 644, radio fluxes and limits from the NRAO VLA Sky Survey (NVSS; Condon et al. 1998) are also available to constrain the current SFR.

In the absence of an AGN component, the [O II] equivalent width can be used to estimate the SFR that is unobscured:

$$
\mathrm{SFR}_{[\mathrm{O} \text { II }]}=8.8 \times 10^{-12} L_{B, \odot} \mathrm{EW}[\mathrm{O} \text { II }] M_{\odot} \mathrm{yr}^{-1}
$$

where $L_{B, \odot}$ is the $B$-band luminosity in solar luminosities (Kennicutt 1992; Barbaro \& Poggianti 1997). Among X-ray sources in Abell 644, Abell 3125, and Abell 3128, only A3125-5 and A3128-2 have measurable [O II] emission (M06); however, the implied $\mathrm{SFR}_{[\mathrm{O} \text { II }}$ for both sources is small ( $\left.\lesssim 1 M_{\odot} \mathrm{yr}^{-1}\right)$. For

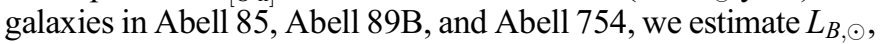
assuming $B-R=1$, which is appropriate for cluster X-ray sources with $[\mathrm{O} \mathrm{II}]$ emission (M06). Among the galaxies with $3 \sigma$ detections of [O $\mathrm{II}]$, two have $\mathrm{SFR}_{[\mathrm{O}}$ II] $>5 M_{\odot} \mathrm{yr}^{-1}, \mathrm{~A} 85-1$ $\left(26 M_{\odot} \mathrm{yr}^{-1}\right)$ and A89B-5 $\left(7 M_{\odot} \mathrm{yr}^{-1}\right)$. A85-1 is an Sbc galaxy (Paturel et al. 2003) whose peculiar velocity ( -3.2 times the velocity dispersion of Abell 85) suggests it is an infalling galaxy toward the edge of the Abell 85 despite its small projected clustercentric distance $\left(0.15 r_{200}\right)$. A89B-5 is also a late-type galaxy (S?; Paturel et al. 2003) at the edge of Abell 89B (0.73r 200$)$. If the [O II] equivalent widths of A85-1 and A89B-5 are indicative of their SFR, then approximately $60 \%$ and $40 \%$ of their X-ray emission could come from HMXBs. However, our identification of both as spectroscopic AGNs suggests that their [O II] likely includes a considerable AGN component. This would lead to an overestimate of their $\mathrm{SFR}_{[\mathrm{O} \text { II] }}$ and implied HMXB X-ray luminosity.

For galaxies without $3 \sigma$ detections of [O II], we conservatively adopted 3 times the measurement error of [O II]. We have excluded A754-7 because its spectra had a low $\mathrm{S} / \mathrm{N}$. Only one remaining galaxy had a large implied $\mathrm{SFR}_{[\mathrm{O}} \mathrm{II}, \mathrm{A} 89 \mathrm{~B}-2\left(<8.8 M_{\odot} \mathrm{yr}^{-1}\right)$. A89B-2 was already excluded as an X-ray AGN due to the expected X-ray emission from diffuse gas and the large offset between the X-ray and optical positions; however, HMXBs could account for $30 \%$ of the X-ray emission from A89B-2. From the combined detections and limits on the SFR from [O II], we conclude that unobscured star formation is not likely to be responsible for the X-ray emission used to identify our X-ray AGNs.

Since [O II] emission can be obscured, one must also consider wavelengths where obscuration is less of an issue. In the farinfrared (FIR), reradiating dust reveals obscured star formation. If one considers the far-infrared SFR relation (Kennicutt 1998), corrected to the IRAS bands (Calzetti et al. 2000), the obscured SFR can be estimated from

$$
\mathrm{SFR}_{\mathrm{FIR}} \approx 7.9 \times 10^{-44} \frac{L_{\mathrm{FIR}}}{\operatorname{ergs~s}^{-1}} M_{\odot} \mathrm{yr}^{-1}
$$

where $L_{\mathrm{FIR}}$ is calculated from the luminosity distance, $D_{L}$, and the IRAS 60 and $100 \mu \mathrm{m}$ fluxes in janskys:

$$
L_{\mathrm{FIR}}=4 \pi D_{L}^{2} 1.26 \times 10^{-14}\left(2.58 F_{60}+F_{100}\right) .
$$

Only two of the X-ray-detected galaxies are detected by IRAS, A85-1 and A754-6, both X-ray AGNs. For A85-1, there are detections at both 60 and $100 \mu \mathrm{m}$, while A754-6 is only detected at $60 \mu \mathrm{m}$. Their predicted $\mathrm{SFR}_{\mathrm{FIR}}$ of about 9 and $<16 M_{\odot} \mathrm{yr}^{-1}$, respectively, imply that approximately $20 \%$ and $<50 \%$ of their $\mathrm{X}$-ray emission could come from HMXBs. The hard median X-ray energy of A754-6 suggests that whatever source is emitting X-rays is obscured; an obscured AGN would also reradiate in the FIR. Although some star formation might be ongoing in these two sources, their identification as X-ray AGNs appears secure.

Given the typical minimum fluxes of detected sources, $F_{60} \sim$ $0.2 \mathrm{Jy}$ and $F_{100} \sim 1 \mathrm{Jy}$ in the IRAS Faint Source Catalog (Moshir et al. 1990), rough upper limits to the $S_{F R}$ FIR of about $<11,<11$, $<13,<14,<18$, and $<22 M_{\odot} \mathrm{yr}^{-1}$ can be set for FIR-undetected galaxies in Abell 754, Abell 85, Abell 3128, Abell 3125, Abell 644, and Abell 89B, respectively. Since a SFR of $10 M_{\odot} \mathrm{yr}^{-1}$ could account for X-ray luminosities from HMXBs of $10^{41} \mathrm{ergs} \mathrm{s}^{-1}$, current SFR limits from IRAS are too shallow to rule out a $100 \%$ 
HMXB origin of the X-ray emission for three of the X-ray AGNs, A89B-5, A644-2, and A3128-9.

In galaxies without a radio $\mathrm{AGN}$, the radio emission at frequencies below tens of gigahertz can be a direct probe of the current star formation of massive stars $\left(M \geq 5 M_{\odot}\right.$; Condon 1992):

$$
\begin{aligned}
& \mathrm{SFR}_{\mathrm{GHz}} \approx \\
& \quad \frac{L_{\mathrm{GHz}}}{\mathrm{W} \mathrm{Hz}^{-1}}\left[5.3 \times 10^{21}\left(\frac{\nu}{\mathrm{GHz}}\right)^{-0.8}+5.5 \times 10^{20}\left(\frac{\nu}{\mathrm{GHz}}\right)^{-0.1}\right]^{-1},
\end{aligned}
$$

where $L_{\mathrm{GHz}}$ is the radio luminosity measured at frequency $\nu$. The X-ray-detected galaxies that have NVSS counterparts are A85-1 (7.5 mJy), A89B-3 (64.6 mJy), A754-1 (158.7 mJy), A754-2 (3.2 mJy), A754-3 (7.3 mJy), A754-4 (71.3 mJy), and A754-7 (81.2 mJy). Three of the sources, A754-1, A754-4, and A754-7, have multiple NVSS components and have been identified as narrow-angle tail radio AGNs (Zhao et al. 1989). ${ }^{8}$ As such, they are not suitable for placing limits on the SFR, and the extended radio sources unambiguously reveal the presence of AGNs. Since the NVSS counterparts to A89B-3 and A754-2 are offset by 19.4" and $36.1^{\prime \prime}$, respectively, they too are unlikely to be due to star formation. In fact, catalog results from the higher resolution $1.4 \mathrm{GHz}$ FIRST survey (White et al. 1997) indicates that A89B-3 is a narrow-angle tail radio AGN, while no FIRST data for A754-2 are available. Only two of the sources have radio emission that could arise from the core of a radio jet or galactic star formation, A85-1 and A754-3. If the radio emission from A85-1 arises from star formation, its $\mathrm{SFR}_{\mathrm{GHz}} \sim 12 M_{\odot} \mathrm{yr}^{-1}$. However, the joint radio and FIR detection allows calculation of its $q$ parameter (e.g., Condon et al. 1991; Yun et al. 2001). With $q=1.717 \pm 0.096$, A85-1 is well away from the typical value of $q=2.34$ for starforming galaxies (Yun et al. 2001), indicative of a radio excess with a likely AGN origin. This suggests its SFR would be lower than what we measure. Given its additional classification as a spectroscopic AGN, its identification as an X-ray AGN seems robust against the effects of star formation. A754-3, whose detection could be consistent with $11 M_{\odot} \mathrm{yr}^{-1}$ of star formation, was not classified as an X-ray AGN due to its low X-ray luminosity that could be due to LMXBs or diffuse gas. Since A754-3 is an early-type galaxy (E-S0; Paturel et al. 2003) at the center of Abell 754, the radio emission appears more likely to arise from a low-luminosity AGN than from star formation. If star formation is ongoing, X-ray emission from HMXBs could also be responsible for the X-ray emission.

With a detection limit of $2.5 \mathrm{mJy}$, the $1.4 \mathrm{GHz}$ NRAO VLA Sky Survey (NVSS) can place tighter constraints on the current SFR than the FIR. For sources undetected in the NVSS, upper limits to the SFR of about $<3.9,<4.0,<6.5$, and $<7.9 M_{\odot} \mathrm{yr}^{-1}$ can be set for galaxies in Abell 754, Abell 85, Abell 644, and Abell 89B, respectively; Abell 3125 and Abell 3128 are too far south to have been included in the survey. These limits are low enough to rule out a strong HMXB origin of the X-ray emission among our sample of X-ray AGNs undetected by NVSS, A85-5 $(<8 \%)$, A89B-5 ( $<44 \%)$, A644-1 $(<5 \%)$, A644-2 $(<38 \%)$, and A754-6 (<10\%).

From the combined constraints on star formation in our $\mathrm{X}$-ray-detected galaxies, we conclude that star formation is not

\footnotetext{
${ }^{8}$ Only one narrow-angle tail candidate in Abell 754, PGC 025746, is undetected in our X-ray observations (Zhao et al. 1989).
}

likely to be responsible for the X-ray emission used to identify our X-ray AGNs. We also conclude that there is not strong evidence for more than a few highly star-forming galaxies (SFR $\gtrsim$ $\left.10 M_{\odot} \mathrm{yr}^{-1}\right)$ in these clusters.

\subsection{AGN Fraction and Host Galaxy Magnitude}

For $L_{\mathrm{X}, B}>10^{41} \mathrm{ergs} \mathrm{s}^{-1}$ and $M_{R}<-20$, the X-ray-identified AGN fraction, $f_{\mathrm{A}}\left(M_{R}<-20, L_{\mathrm{X}, B}>10^{41}\right)$, summing over galaxies in eight $z \lesssim 0.3$ clusters was $2.2 \%$, and $f_{\mathrm{A}}\left(M_{R}<-21.3\right.$, $\left.L_{\mathrm{X}, B}>10^{41}\right)=9.8 \%$ (M06). Sun et al. (2007) found $f_{\mathrm{A}}\left(M_{R}<\right.$ $\left.-21.3, L_{\mathrm{X}, B}>10^{41}\right) \sim 5 \%$ for $0.01<z<0.05$ clusters. This presents an indication that the optical luminosity of a host galaxy affects whether an X-ray AGN is detected at these X-ray luminosities. In determining these AGN fractions, neither sample eliminated luminous X-ray galaxies whose emission could actually be due to diffuse gas. This may affect the Sun et al. (2007) X-ray AGN sample more, as half of them were in BCGs, while none of the M06 X-ray AGNs were. In the following section we explore the magnitude dependence of $f_{\mathrm{A}}$ for galaxies in our sample, after applying the $L_{\mathrm{X}} / L_{K_{s}}$ relations to select X-ray AGNs.

We calculated the extinction-corrected, absolute $M_{R}$ and $M_{K_{s}, K 20}$ rest-frame magnitudes for all available cluster galaxies, as in $\S 2.2$ and Table 4. Based on all cluster galaxies with both magnitudes, we find the distribution of rest-frame colors $M_{R}-M_{K_{s}}$ is consistent with a Gaussian distribution centered on 2.82 with a dispersion of 0.26 . Since we were unable to determine whether the BCG of Abell 85 contained an X-ray AGN, we removed it from the sample. In the top panels of Figure 5 we compare the $M_{R}$ (left) and $M_{K_{s}}$ (right) distributions of galaxies with AGNs (dashed line) and without AGNs (solid line). The distributions are clearly different; Kolmogorov-Smirnov (K-S) tests indicate that the probabilities they are the same are $1.9 \times 10^{-3}$ and $5.7 \times 10^{-3}$, respectively. In the bottom panels of Figure 5 we display $f_{\mathrm{A}}\left(L_{\mathrm{X}, B}>10^{41}\right)$ in magnitude bins. For the galaxies in Abell 644 and Abell 3125, we applied a correction ( 3.9 and 1.4, respectively; see Table 6 ) to the number of galaxies at a given magnitude to account for their incomplete membership information (M07). This was necessary because membership is incomplete at these optical magnitudes, but any X-ray-detected optical source was always targeted for spectroscopy in M07. The spectroscopic measurements for Abell 85, Abell 89B, and Abell 754 are 100\% complete at $m_{R}<16$, but completeness does drop to $\sim 20 \%-50 \%$ by $m_{R}=18$ (CZ03). We note that $M_{R}=-20$ corresponds to $m_{r} \sim$ 17.5 for Abell 89B and $m_{r} \sim 16.9$ for Abell 85 and Abell 754 . Since these measurements were made prior to our analysis of the X-ray data, the spectroscopic completeness should be largely independent of the X-ray properties of galaxies; any completeness correction would equally correct the numerator and denominator in the fractions involved. Although one might be concerned that AGNs with emission lines are more likely to have a measured redshift, we note that only a small fraction of X-ray AGNs $(\sim 20 \%)$ have such emission lines. Any correction for such an effect would be smaller than the current error bars on AGN fractions, which are limited by the small numbers of AGNs. We further note that we found no X-ray source matched to a photometric object without a redshift that would be consistent with $M_{R}<-20$.

Since it is difficult to construct a clear situation in which the X-ray completeness depends on the optical/near-IR host magnitude of the galaxy, we do not expect that sources not detected as $\mathrm{X}$-ray AGNs due to X-ray incompleteness are the cause of this discrepancy. To explicitly test this, we considered two sets of X-ray AGNs at brighter luminosities: X-ray AGNs with $L_{\mathrm{X}, B}>$ $4 \times 10^{41} \mathrm{ergs} \mathrm{s}^{-1}$ over the entire sample and X-ray AGNs with $L_{\mathrm{X}, B}>2 \times 10^{41} \mathrm{ergs} \mathrm{s}^{-1}$ when removing all A89B galaxies from 

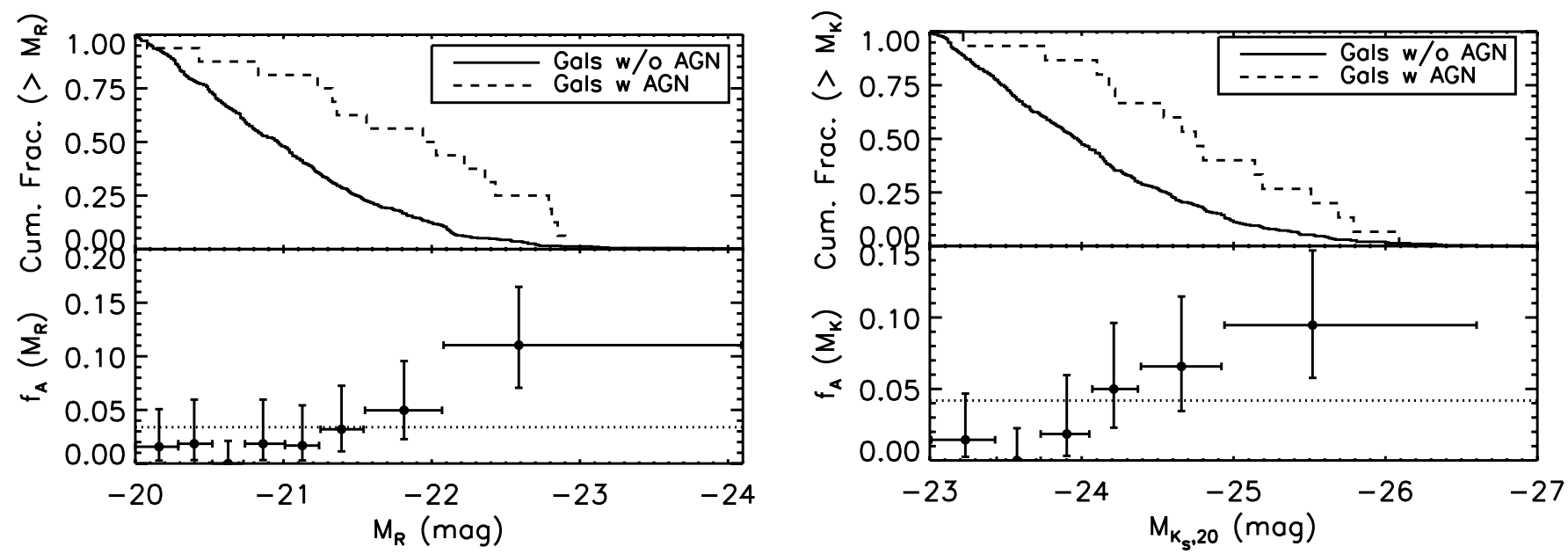

FIG. 5.-Top: Cumulative fraction of galaxies with (dashed line) and without (solid line) X-ray AGNs as a function of $M_{R}<-20$ (left) and $M_{K}, K 20<-23.0($ right) for galaxies in the cluster sample from Table 3. Bottom: The fraction of galaxies with an X-ray AGN, $f_{\mathrm{A}}$, in bins of approximately 50 galaxies. In both panels the dotted line indicates the fraction summing over all $M_{R}<-20$ galaxies in the sample, $3.4_{-0.8}^{+1.1} \%$. It is clear that more luminous galaxies are more likely to contain X-ray AGNs.

consideration. In both cases we still find that the optical/near-IR magnitudes of galaxies with and without X-ray AGNs are drawn from different distributions using the K-S test.

Since the X-ray emission from LMXBs and diffuse gas also increases with optical/near-IR magnitude, one concern is that the higher fractions of X-ray AGNs at brighter magnitudes is due to normal X-ray-emitting galaxies that are misidentified as X-ray AGNs. To address this, we have performed Monte Carlo simulations to determine how severely our AGN sample could be contaminated by the combination of X-ray emission from LMXBs and diffuse gas.

We performed $10^{5}$ realizations of the X-ray to near-IR luminosity relations for both LMXBs (eq. [1]) and diffuse gas (eq. [3], with proper corrections), assuming that the reported errors in the relations follow Gaussian statistics. Within each realization, we combined the two simulated relations to predict the X-ray luminosity from LMXBs and diffuse gas for each cluster galaxy. Since our measured luminosities were for point sources, while the predicted luminosities were for entire galaxies, we needed to determine and apply a correction factor. Comparisons of the point-source counts and the counts within the $K_{s}=20 \mathrm{mag} \operatorname{arcsec}^{2}$ isophote for the $\mathrm{X}$-ray-detected galaxies not classified as X-ray AGNs indicated that the average luminosity of the entire galaxy was 1.5 times that for a point source. We applied this correction to the predicted luminosities and then converted to an expected number of counts for each realization assuming an exposure time appropriate for the flanking-field observations. This expected number of counts was used to create a simulated output number of counts assuming random deviations drawn from Poisson distributions with the expected number of counts as its mean. The output number of counts and its error were then converted back into luminosity, $L_{\mathrm{X}, B, M C}$, and we performed the same (luminosity) selection criteria to identify a source as an X-ray AGN: $L_{\mathrm{X}, B, M C}>10^{41} \mathrm{erg} \mathrm{s}^{-1}$ and $L_{\mathrm{X}, B, M C}$ more than $1 \sigma$ higher than the sum of the upper limits to the Kim \& Fabbiano (2004) and Sun et al. (2007) relations. Thus, for each cluster galaxy we could calculate the probability that a source was misidentified as an X-ray AGN, $p_{\text {false }}$ (Fig. 6).

For $M_{K_{s}} \gtrsim-24.8, p_{\text {false }} \lesssim 0.02$ and drops as host galaxy optical luminosity decreases due to the $10^{41} \mathrm{ergs} \mathrm{s}^{-1}$ minimum X-ray luminosity for X-ray AGN classification. For $M_{K_{s}} \lesssim-25.2, p_{\text {false }} \lesssim$ 0.01 and roughly drops as host galaxy optical luminosity increases. This is due to the X-ray AGN selection criteria set by the X-ray emission expected from LMXBs and gas. The roughly diagonal line up to $M_{K_{s}} \gtrsim-24.8$ corresponds to a minimum of 5 counts. Similar rough diagonal lines at increasing galaxy optical luminosity corresponds to an increasing minimum number of counts

TABLE 6

AGN FraCtion

\begin{tabular}{|c|c|c|c|c|c|c|}
\hline \multirow[b]{2}{*}{$\begin{array}{c}\text { Cluster Name } \\
\text { (1) }\end{array}$} & \multicolumn{2}{|c|}{$N_{\mathrm{XAGN}}\left(M_{R}<-20\right)$} & \multicolumn{2}{|c|}{ Members $\left(M_{R}<-20\right)$} & \multicolumn{2}{|c|}{$f_{\mathrm{A}}$} \\
\hline & $\begin{array}{c}L_{\mathrm{X}, B}>10^{41} \\
\quad \text { (2) }\end{array}$ & $\begin{array}{c}L_{\mathrm{X}, B}>10^{42} \\
\text { (3) }\end{array}$ & $\begin{array}{l}\text { Confirmed } \\
\text { (4) }\end{array}$ & $\begin{array}{l}\text { Corrected } \\
\text { (5) }\end{array}$ & $\begin{array}{c}L_{\mathrm{X}, B}>10^{41} \\
\text { (6) }\end{array}$ & $\begin{array}{c}L_{\mathrm{X}, B}>10^{42} \\
\text { (7) }\end{array}$ \\
\hline Abell 85 .. & 2 & 0 & 109 & 109 & $0.018_{-0.012}^{+0.024}$ & $0.000_{-0.000}^{+0.010}$ \\
\hline 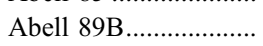 & 2 & 1 & 22 & 22 & $0.091_{-0.058}^{+0.012}$ & $0.045_{-0.038}^{+0.0007}$ \\
\hline Abell $3125 \ldots \ldots \ldots \ldots \ldots \ldots$ & 3 & 0 & 20 & 28 & $0.107_{-0.058}^{+0.093}$ & $0.000_{-0.000}^{+0.048}$ \\
\hline 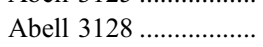 & 4 & 1 & 67 & 67 & $0.060_{-0.028}^{+0.058}$ & $0.015_{-0.012}^{+0.000}$ \\
\hline Abell 754 & 3 & 1 & 171 & 171 & $0.018_{-0.010}^{+0.028}$ & $0.006_{-0.005}^{+0.012}$ \\
\hline Abell 644 & 2 & 1 & 19 & 75 & $0.027_{-0.017}^{+0.010}$ & $0.013_{-0.011}^{+0.005}$ \\
\hline 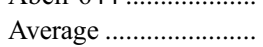 & & & & & $0.031_{-0.011}^{+0.017}$ & $0.009_{-0.006}^{+0.011}$ \\
\hline Sum & 16 & 4 & 408 & 472 & $0.034_{-0.008}^{+0.011}$ & $0.008_{-0.004}^{+0.006}$ \\
\hline
\end{tabular}

Notes.-AGN fractions for $M_{R}<-20$ galaxies in six $z<0.08$ clusters. Col. (1): Cluster name. Col. (2): Number of X-ray AGNs with $L_{\mathrm{X}, B}>10^{41} \mathrm{ergs} \mathrm{s}^{-1}$. (3) Number of X-ray AGNs with $L_{\mathrm{X}, B}>10^{42} \mathrm{ergs} \mathrm{s}^{-1}$. Col. (4): Number of $M_{R}<-20$ galaxies with spectroscopically confirmed redshifts within Chandra FOV. Col. (5): Number of $M_{R}<-20$ galaxies within Chandra FOV, corrected for preferential spectroscopic targeting of X-ray detections. Col. (6): X-ray AGN fraction with $L_{\mathrm{X}, B}>10^{41} \mathrm{ergs} \mathrm{s}^{-1}$; (7) X-ray AGN fraction with $L_{\mathrm{X}, B}>10^{42} \mathrm{ergs} \mathrm{s}^{-1}$. 


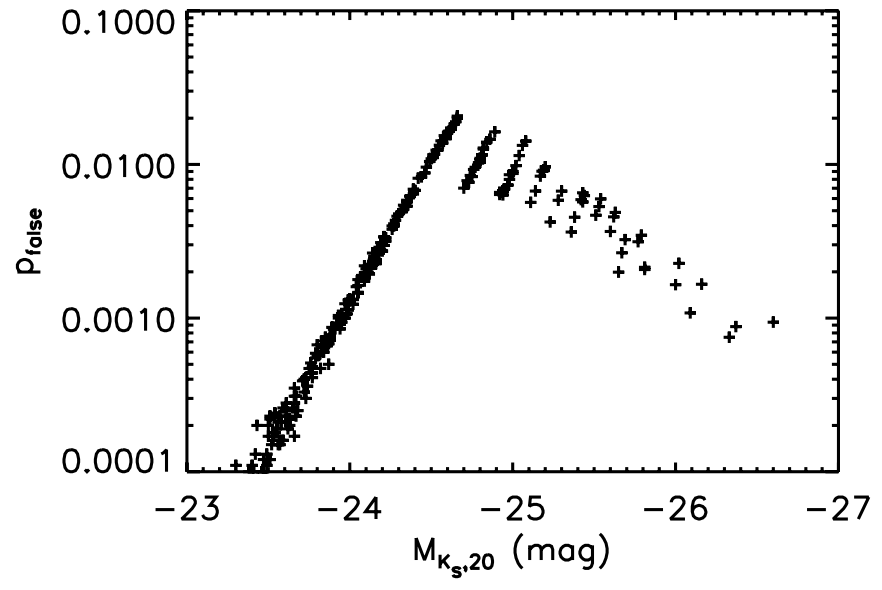

FIG. 6.- Probability that a cluster galaxy would be falsely identified as an $\mathrm{X}$-ray AGN as a function of $M_{K_{s} K 20}$. For the vast majority of cluster galaxies the probability that X-ray emission from LMXBs and diffuse gas would be misclassified as an X-ray AGN is small. The total number of falsely identified AGNs over the entire sample is $<1.1$.

that satisfy our criteria. As the exposure times are made larger, as in our central field observations, the values of $p_{\text {false }}$ drop at a given $M_{K_{s}}$. Therefore, calculations based on Figure 6 represent the most conservative, i.e., highest, estimate of the number of sources in our entire sample that are falsely identified as X-ray AGNs due to their LMXB and diffuse gas emission. By summing the probabilities, we estimate that there are $<1.1$ such false sources. Approximately $0.3,0.6$, and 0.2 falsely identified sources are predicted for the three brightest bins, respectively, in each of the bottom panels of Figure 5. This would reduce their fractions by $\lesssim 0.005,0.010$, and 0.003 , which is much less than the current error bars. In addition, we note that even after removing the three AGNs closest to the expected relation combining LMXBs and diffuse gas, the K-S test still indicates that the $M_{R}(l e f t)$ and $M_{K_{\mathrm{s}}}$ (right) distributions of galaxies with and without AGNs are not drawn from the same distributions. We conclude that misidentified X-ray emission from LMXBs and diffuse gas are not responsible for X-ray-detected AGNs being more likely to be found in more luminous galaxies.

Our results that X-ray-detected AGNs are more likely to be found in more luminous galaxies for $M_{R}$ are consistent with results from the XMM-Newton detections of AGNs in the Abell 901/902 supercluster $(z \sim 0.17$; Gilmour et al. 2007). Both show a nearly constant fraction of X-ray AGNs, of $\sim 1 \%$, for $-21.5 \lesssim M_{R} \lesssim$ -20 galaxies. For brighter galaxies, this increases to a maximum of $\sim 10 \%-20 \%$. We can use $M_{K_{s}, K 20}$ to derive galactic stellar masses for these galaxies. If we use Bruzual \& Charlot (2003), assuming their Padova 1994 evolutionary tracks for a 13 Gyr, solar abundance single stellar population with the Chabrier (2003) initial mass function, and correct the isophotal magnitude to a total magnitude, the magnitudes in Figure 5 (right) correspond from about $5 \times 10^{10}$ to $2 \times 10^{12} M_{\odot}$. A similar trend has been observed for radio-loud AGNs, for which the fraction of radio-loud AGNs increases with stellar mass, $f \propto M_{*}^{2.5}$ up to $\sim 10^{12} M_{\odot}$ (Best et al. 2005). On the other hand, the fraction of strong $\left(L[\mathrm{O} \mathrm{III}]>10^{7} L_{\odot}\right)$, optically identified AGNs drops from $\sim 12 \%$ at $10^{11} M_{\odot}$ to $\sim 2 \%$ at $10^{10}$ and $10^{12} M_{\odot}$ (Kauffmann et al. 2003b). The physically relevant question that these trends raise is whether AGNs are more likely to reside in more luminous (massive) host galaxies.

In the case of our X-ray AGNs, much of the signal may actually be due to a physical selection bias. For each of our X-ray AGNs, we can roughly estimate the accretion rate relative to the

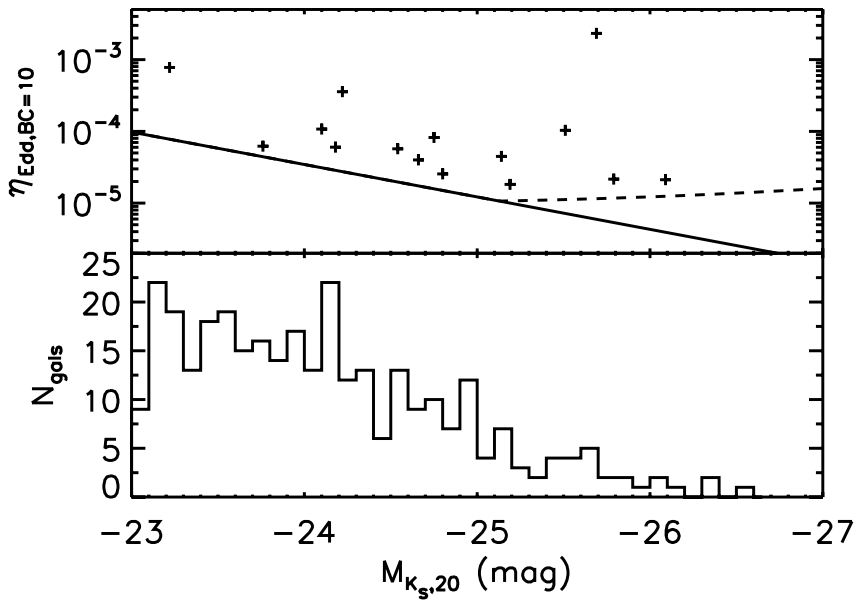

Fig. 7.-Top: Approximate Eddington-normalized accretion rates, $\eta_{\text {Edd }}$, of detected X-ray AGNs as a function of $M_{K_{s} K 20}$. We assume a bolometric correction to the X-ray luminosity, $\mathrm{BC}=10$. Since we assumed all galaxies are spheroids, their black hole mass may be lower and the normalized accretion rates may be higher at a given $M_{K_{s} K 20}$. The solid line indicates the limit set by $L_{\mathrm{X}, B}>$ $10^{41} \mathrm{ergs} \mathrm{s}^{-1}$. The dashed line indicates the limit set to exclude potential contributions from LMXBs and diffuse gas. The changing AGN fraction with host galaxy luminosity (Fig. 5) is likely due to a selection bias where lower Eddington accretion efficient sources are only detectable in more luminous galaxies, which are more likely to have more massive black holes. Bottom: The histogram of $M_{K_{s} K 20}$ cluster member galaxies is shown for reference.

Eddington value, divided by the bolometric correction, $\eta_{\mathrm{Edd}} / \mathrm{BC}$, from $L_{\mathrm{X}, B}$. As near-IR light is a better tracer of mass and subject to less extinction than visible light, we converted the detected near-IR luminosity to a black hole mass (Marconi \& Hunt 2003) under the assumption that all the luminosity is from a bulge component, $\log M_{\mathrm{BH}}=8.21+1.13\left(\log L_{K_{s}, \text { tt }}\right)$. Since these sources are likely to have a range of disk contributions to their luminosities, our derived $\eta_{\text {Edd }} / \mathrm{BC}$ are underestimated; however, our results are still illustrative. Although $\mathrm{BC}$ is uncertain, especially if the mechanism for low- and high-luminosity $\mathrm{X}$-ray AGNs differ, we assume $\mathrm{BC}=10$ and display $\eta_{\mathrm{Edd}, \mathrm{BC}=10}$ in Figure 7. This assumption is reasonable given derived values of $\mathrm{BC}$ at these $\mathrm{X}$-ray luminosities (in the $2-10 \mathrm{keV}$ band; Vasudevan \& Fabian 2007). The detected sources cover ranges of $1 \times 10^{-5} \lesssim \eta_{\text {Edd, BC }=10} \lesssim$ $3 \times 10^{-3}$. Given our requirement that $L_{\mathrm{X}, B}>10^{41} \mathrm{ergs} \mathrm{s}^{-1}$, we can calculate the minimum $\eta_{\mathrm{Edd}, \mathrm{BC}=10}$. It is clear that at higher luminosities, X-ray AGNs at lower $\eta_{\mathrm{Edd}, \mathrm{BC}=10}$ can be, and are, detected. Similar results are also seen in Abell 901/902 (Gilmour et al. 2007); their $\eta_{\text {Edd }}$ values are $\sim 5$ higher because they adopted the $R$-band host-galaxy luminosity black hole mass relation of McLure \& Dunlop (2002). Calculated AGN fractions will only be independent of the galaxy luminosity function when they are sensitive to the same $\eta_{\text {Edd }}$.

Since more massive galaxies tend to be more centrally concentrated (e.g., Kauffmann et al. 2003a), indicative of a more dominant bulge component, our $\eta_{\mathrm{Edd}, \mathrm{BC}=10}$ values are likely to represent larger underestimates at lower near-IR luminosities than at higher luminosities. Thus, the disparity between the $\eta_{\mathrm{Edd}, \mathrm{BC}=10}$ probed by a given X-ray luminosity at lower and higher near-IR luminosities is even larger than that suggested by Figure 7 .

There are two implication of this selection bias. First, this bias makes it essential that comparisons of AGN fractions make the same assumptions in both their X-ray luminosity and optical magnitude cuts, and that host galaxy morphology (spheroid mass) may also be important. More importantly, $\eta_{\mathrm{Edd}} / \mathrm{BC}$ is a more physical measure of AGN activity than the X-ray luminosity. The true 

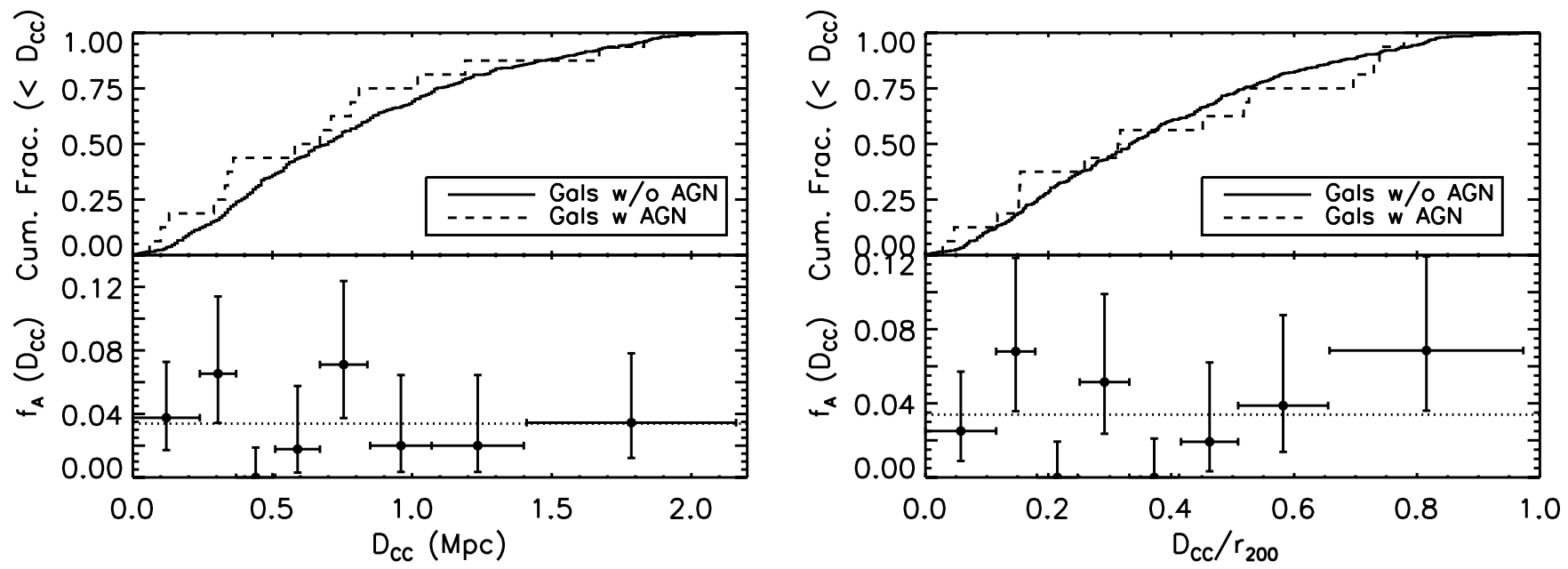

FIG. 8.-Top: Cumulative fraction of $M_{R}<-20$ galaxies with (dashed line) and without (solid line) X-ray AGNs as a function of clustercentric distance, $D_{\mathrm{CC}}$, in $\mathrm{Mpc}($ left $)$ and units of $r_{200}$ (right) for galaxies in the cluster sample from Table 3. Bottom: Fraction of galaxies with an X-ray AGN, $f_{\mathrm{A}}$, in bins of approximately 50 galaxies. The dotted line indicates the fraction for the $M_{R}<-20$ sample. The radial distributions of galaxies with and without an X-ray AGNs are comparable, consistent with M07 results for $L_{\mathrm{X}, B}>10^{41} \mathrm{ergs} \mathrm{s}^{-1}$ and Ruderman \& Ebeling (2005) results for disturbed clusters.

fraction of $M_{R}<-20$ galaxies that host X-ray AGNs with $\eta_{\text {Edd }} /$ $\mathrm{BC} \gtrsim 10^{-6}$ will be larger than the $\sim 3 \%$ we measure.

\subsection{Radial Distribution of AGNS}

One of the goals of the new observations of Abell 85 and Abell 754 was to measure the spatial distribution of X-raydetected AGNs. Along with Abell 89B, these three clusters have partial coverage out to $\sim r_{200}$. Although the radial coverage of the other three clusters is smaller, we include them in our measurement of the radial distribution. The Chandra observations of Abell 644 and Abell 3128 have coverage out to $\sim 0.4 r_{200}$, while the one of Abell 3125 extends farther, to $\sim 0.7 r_{200}$. Since we never include galaxies outside of the Chandra FOVs, including all six clusters will improve the statistical determination of the AGN fraction with radius, particularly for smaller radii.

In the top panels of Figure 8, we compare the (projected) spatial distributions of $M_{R}<-20$ galaxies with AGNs (dashed line) and without AGNs (solid line). We consider the distributions as a function of physical distance (left) and distance scaled to $r_{200}$ (right). In the bottom panels of Figure 8 we display $f_{\mathrm{A}}\left(M_{R}<\right.$ $\left.-20, L_{\mathrm{X}, B}>10^{41}\right)$ in spatial bins, correcting for membership completeness. For this sample of galaxies, the spatial distributions of galaxies with X-ray AGNs are the same as galaxies without X-ray AGNs.

As X-ray incompleteness is only a minor issue at the edges of the flanking-field observations of Abell 85 and Abell 754 and the single observation of Abell 3125, we do not believe it is masking an increased contribution of AGNs at the outskirts of clusters. The upper error bars presented in any single bin in the bottom panels of Figure 8 correspond to roughly one to three missing sources in a given bin. Since the edges of the flanking fields cover a wide range of clustercentric distances, any missing sources should be spread over multiple bins. Thus, the effect from missing only one or two sources over the entire sample is well within our current noise level. In addition, we do not find any radial dependence in the smaller samples of AGNs where we are complete: $L_{\mathrm{X}, B}>$ $4 \times 10^{41} \mathrm{ergs} \mathrm{s}^{-1}$ in the entire sample or $L_{\mathrm{X}, B}>2 \times 10^{41} \mathrm{ergs} \mathrm{s}^{-1}$ when removing all $\mathrm{A} 89 \mathrm{~B}$ galaxies from consideration.

In M07 the spatial distribution of $L_{\mathrm{X}, B}>10^{41} \mathrm{ergs} \mathrm{s}^{-1} \mathrm{X}$-ray AGNs was also consistent with the other cluster members. We do note that our and their distribution are not independent, as they share Abell 644, Abell 3125, and 3128. There are too few
X-ray AGNs with $L_{\mathrm{X}, B}>10^{42} \mathrm{ergs} \mathrm{s}^{-1}$ in our sample to test their result that the more X-ray luminous AGNs are more centrally concentrated.

One concern is that galaxies with a small projected distance may actually be infalling galaxies at large physical distances close to the line of sight to the cluster. In Figure 9 we plot the infall velocity relative to the mean cluster velocity scaled by the cluster velocity dispersion against the projected distance scaled to $r_{200}$ for $M_{R}<-20$ galaxies with AGNs (circles) and without AGNs (diamonds). Although there are two AGNs with large infall velocities at small projected distances, A85-1 and A644-2, a two-dimensional K-S test (Press et al. 1992) indicates that the galaxies with and without AGNs are not likely to be drawn from samples with different two-dimensional distributions.

If AGNs are fueled by galaxy-galaxy interactions, one expects AGNs should be more prevalent in the outskirts of clusters. However, a significant fraction of early type galaxies, which tend to lie in the centers of richest clusters, are known to harbor lowluminosity AGNs and LINERs. A relation between AGNs and

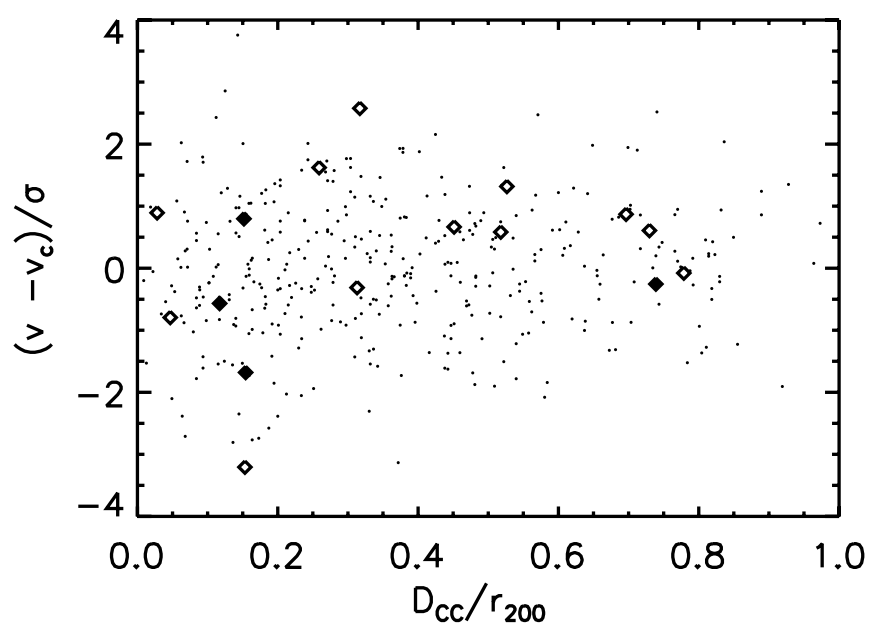

FIG. 9.- Radial velocities of cluster $M_{R}<-20$ galaxies relative to the mean velocity of each cluster and normalized by the cluster velocity dispersion as a function of cluster-centric distance, $D_{\mathrm{CC}}$, in units of $r_{200}$. Diamonds indicate galaxies detected as X-ray AGNs. Filled diamonds indicate $L_{\mathrm{X}, B}>10^{42} \mathrm{ergs} \mathrm{s}^{-1}$. The two-dimensional distributions of galaxies with and without an X-ray AGN are comparable. 
early-type galaxies could dilute or even reverse the trends predicted by gas-rich mergers or galaxy harassment. In addition, our detected X-ray AGNs are detected more often in more luminous host galaxies. Since more massive galaxies tend to be early-type galaxies, any observed radial trend due to low-luminosity AGNs and LINERs should be larger than it is intrinsically. The combination of all three effects could explain why the radial distribution of X-ray AGNs is not markedly different from that of all galaxies in our sample. Another potential explanation is that radial averaging over the substructure in the cluster masks any underlying signal. Finally, there could actually be no preferred radial distribution for X-ray AGNs.

\section{AGN FRACTION AND CLUSTER PROPERTIES}

M07 found some evidence that the AGN fraction varies with the properties of the host cluster. When summing over galaxies, their $f_{\mathrm{A}}\left(M_{R}<-20, L_{\mathrm{X}, B}>10^{41}\right)$ is $2.2 \%$; however, their cluster-averaged $f_{\mathrm{A}}\left(M_{R}<-20, L_{\mathrm{X}, B}>10^{41}\right)$ is $4.9 \%$. Compared to the cluster-by-cluster $f_{\mathrm{A}}\left(M_{R}<-20, L_{\mathrm{X}, B}>10^{41}\right)$, a $\chi^{2}=17.8$ for 7 degrees of freedom (dof) indicates that cluster variations of the AGN fraction are significant. They find weak evidence that the AGN fraction is higher at lower redshift, in lower velocity-dispersion clusters, in clusters with substantial substructure, and in clusters with a smaller fraction of ButcherOemler galaxies. However, they caution that correlations between several of these parameters preclude identification of which cluster property or properties most strongly influence the AGN fraction.

In Table 6 we summarize the AGN fractions for each cluster in our sample. We first list the number of X-ray AGNs more luminous than $10^{41} \mathrm{ergs} \mathrm{s}^{-1}$ (col. [2]) and $10^{42} \mathrm{ergs} \mathrm{s}^{-1}$ (col. [3]). We then list the number of spectroscopically confirmed, $M_{R}<$ -20 members in each cluster (col. [4]). Since some of the galaxies within M06 clusters were preferentially targeted for spectroscopy based on X-ray detections and completeness for membership of two of the clusters (Abell 644 and Abell 3125) is not 100\%, we also list a corrected number of $M_{R}<-20$ galaxy members in each cluster (col. [5]). We use these numbers to calculate the X-ray AGN fraction above $10^{41} \mathrm{ergs} \mathrm{s}^{-1}$ (col. [6]) and $10^{42} \mathrm{ergs} \mathrm{s}^{-1}$ (col. [7]). As in M07, we also calculate the cluster-by-cluster average AGN fractions, and the AGN fraction assuming it is independent of cluster properties. We find that the AGN fractions summing over galaxies, $f_{\mathrm{A}}\left(M_{R}<-20, L_{\mathrm{X}, B}>10^{41}\right)=3.1 \% \pm$ $1.1 \%$ and $f_{\mathrm{A}}\left(M_{R}<-20, L_{\mathrm{X}, B}>10^{42}\right)=0.9 \% \pm 0.6 \%$, are consistent with the cluster-averaged AGN fractions $f_{\mathrm{A}}\left(M_{R}<-20\right.$, $\left.L_{\mathrm{X}, B}>10^{41}\right)=3.4_{-0.8}^{+1.1} \%$ and $f_{\mathrm{A}}\left(M_{R}<-20 ; L_{\mathrm{X}, B}>10^{42}\right)=$ $0.8_{-0.4}^{+0.7} \%$. Comparing $f_{\mathrm{A}}\left(M_{R}<-20, L_{\mathrm{X}, B}>10^{41}\right)$ of each cluster to the cluster-averaged AGN fraction, $\chi^{2}=4.8$ for 5 dof. We note that these numbers have not been corrected for the one or two sources that may be missed due to X-ray incompleteness. This change is minimal, as the upper error bars (due to the small number of X-ray AGNs) correspond to the roughly two to three missing sources for any given cluster, and roughly five sources for the entire sample.

With our current data for Abell 85, Abell 89, and Abell 754, we choose to concentrate in the paragraphs below on the relation between AGN fraction and two cluster properties, redshift and velocity dispersion. With the narrow redshift but wide velocitydispersion range, we have a greater ability to break the degeneracy between the two that was present in M07.

\subsection{AGN Fraction and Redshift}

Since our sample spans a narrow redshift range, we consider other samples to test whether there is any redshift evolution of the X-ray AGN fraction. To compare the AGN fractions we determine to those in M07, the overlapping clusters Abell 644, Abell 3125, and Abell 3128 must be removed from M07. The remaining five clusters form a $0.15<z<0.32$ sample. In this sample, the AGN fractions summing over galaxies, $f_{\mathrm{A}}\left(M_{R}<-20\right.$, $\left.L_{\mathrm{X}, B}>10^{41}\right)=1.4_{-0.3}^{+0.4} \%$ and $f_{\mathrm{A}}\left(M_{R}<-20, L_{\mathrm{X}, B}>10^{42}\right)=$ $0.8_{-0.3}^{+0.4} \%$, are consistent with the cluster-averaged AGN fraction $f_{\mathrm{A}}\left(M_{R}<-20, L_{\mathrm{X}, B}>10^{41}\right)=1.2 \% \pm 0.5 \%$ and $f_{\mathrm{A}}\left(M_{R}<\right.$ $\left.-20, L_{\mathrm{X}, B}>10^{42}\right)=0.9 \% \pm 0.4 \%$. One might then conclude that the AGN fraction at lower redshifts is indeed higher based on $f_{\mathrm{A}}\left(M_{R}<-20, L_{\mathrm{X}, B}>10^{41}\right)$, as suggested in M07; however, M07 also note that most of the higher redshift sample is not $\mathrm{X}$-ray complete down to $10^{41} \mathrm{ergs} \mathrm{s}^{-1}$. At $L_{\mathrm{X}, B}>10^{42} \mathrm{ergs} \mathrm{s}^{-1}$, where the sample should be complete, there is no evidence for redshift evolution in $f_{\mathrm{A}}\left(M_{R}<-20, L_{\mathrm{X}, B}>10^{42}\right)$. We also compare our AGN fraction to that of the $0.01<z<0.05$ sample of Sun et al. (2007). We measure $f_{\mathrm{A}}\left(M_{R}<-21.3, L_{\mathrm{X}, B}>10^{41}\right)=$ $6.7_{-1.9}^{+2.4} \%$, while Sun et al. (2007) find $f_{\mathrm{A}}\left(M_{R}<-21.3, L_{\mathrm{X}, B}>\right.$ $\left.10^{41}\right)=5.5_{-1.8}^{+2.4} \%$. Thus, we believe that there is no measurable redshift evolution in the X-ray AGN fraction for $z \leqq 0.3$ in the current samples. As the Sun et al. (2007) sample is of more nearby clusters, it samples a more centrally concentrated population of galaxies than the sample in this paper. Due to the combination of our errors being limited by small numbers of AGNs and our result that there is no preferred radial distribution of AGNs, this mismatch is not likely to play a large role in this conclusion.

Recently, a large fraction of luminous X-ray AGNs in $z \sim 0.6$ clusters was measured (Eastman et al. 2007). Due to the redshift of these clusters and the sensitivity of the observations, fractions were measured for hard (2.0-10.0) band X-ray luminosities, $L_{\mathrm{X}, H}$ above $10^{42}$ and $10^{43} \mathrm{ergs} \mathrm{s}^{-1}$. Eastman et al. find $f_{\mathrm{A}}\left(M_{R}<\right.$ $\left.-20, L_{\mathrm{X}, H}>10^{42}\right)=2.8_{-1.0}^{+1.5} \%$ and $f_{\mathrm{A}}\left(M_{R}<-20, L_{\mathrm{X}, H}>\right.$ $\left.10^{43}\right)=2.0_{-0.7}^{+1.0} \%$, and that these fractions were $\sim 20$ times that of $z \sim 0.2$ clusters, which is much larger than the expected increases, factors of 1.5 and 3.3, from the measured evolution of the field AGN space density (Ueda et al. 2003). The largest statistical uncertainty came from the lower redshift sample. Although we note that there are issues left to explore regarding the evolution of the AGN fraction in clusters (e.g., the $z \sim 0.6$ clusters are not necessarily the progenitors of the $z \sim 0.2$ clusters; $M_{R}^{*}$ is $\sim 0.4$ brighter at $z \sim 0.6$ than at $z \sim 0.2$ ), we can add the results of Abell 85, Abell 89B, and Abell 754 to Martini et al. (2007) to refine the estimate for $z \sim 0.2$ clusters. In the hard band, A754-1 is $\sim 9.9 \times 10^{42} \mathrm{ergs} \mathrm{s}^{-1}$ before pileup corrections. These corrections are likely to make it more luminous than $10^{43} \mathrm{ergs} \mathrm{s}^{-1}$. No other AGNs in these clusters has $L_{\mathrm{X}, H}>10^{42} \mathrm{ergs} \mathrm{s}^{-1}$. Thus, for $z \sim 0.2$ clusters, we find $f_{\mathrm{A}}\left(M_{R}<-20, L_{\mathrm{X}, H}>10^{42}\right)=$ $0.18_{-0.10}^{+0.17} \%$ and $f_{\mathrm{A}}\left(M_{R}<-20, L_{\mathrm{X}, H}>10^{43}\right)=0.12_{-0.08}^{+0.16 \%}$. These fractions are consistent with the fractions reported in Eastman et al. (2007), but with smaller confidence intervals. We note that the increased spatial coverage provided by the clusters in this paper also provides a better match to the more distant clusters, which are sampled out to their projected $r_{200}$.

\subsection{AGN Fraction and Velocity Dispersion}

The lack of a radial dependence of X-ray AGN fraction in our sample may be due to the true absence of a trend or the masking of the expected increasing trend with radius by several other factors, including our increased sensitivity to AGNs in massive galaxies, which tend to lie in cluster cores, and the significant population of known low-luminosity AGNs/LINERs in early-type galaxies, which are also more numerous in cluster cores. Any trend might also have been diluted by our averaging over any 


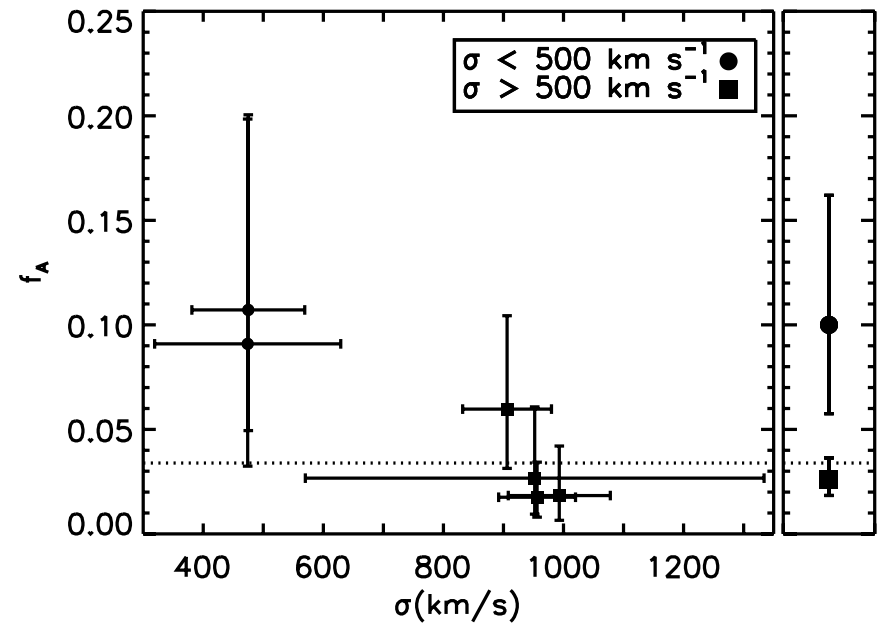

FIg. 10. - Left: Fraction of $M_{R}<-20$ galaxies with X-ray AGNs, $f_{\mathrm{A}}$, vs. cluster velocity dispersion in the cluster sample from Table 3 . The dotted line indicates the fraction summing over all $M_{R}<-20$ galaxies in the sample. Right: Values of $f_{\mathrm{A}}$ summing over galaxies in clusters with velocity dispersions under and over $500 \mathrm{~km} \mathrm{~s}^{-1}$ are indicated with large symbols. The clusters with the lowest velocity dispersion have the highest fractions, consistent with a preference for X-ray AGNs in the regions with a grouplike environments from the Abell 901/902 supercluster (Gilmour et al. 2007).

substructures at a given radius. With better AGN statistics, it may be possible to consider whether the AGN fraction increases in grouplike substructures in the cluster relative to the cluster core, a truer test of the hypothesis that mergers drive AGNs today. For now, we employ another test of the effect of environment on AGN fraction and thus of the merger scenario: is there a change in X-ray AGN fraction as the velocity dispersions of clusters increase?

We display $f_{\mathrm{A}}\left(M_{R}<-20, L_{\mathrm{X}, B}>10^{41}\right)$ as a function of cluster velocity dispersion for our $z \lesssim 0.08$ cluster sample in Figure 10. In our sample, comparing the AGN fraction of each cluster to the cluster-averaged AGN fraction does not indicate a strong variation. However, we find a correlation between AGN fraction and velocity dispersion. Clusters with lower velocity dispersion have larger $f_{\mathrm{A}}\left(M_{R}<-20, L_{\mathrm{X}, B}>10^{41}\right)$ in our data. In particular, the two clusters with the highest AGN fraction, Abell 89B and Abell 3125 have velocity dispersions of $\sim 500 \mathrm{~km} \mathrm{~s}^{-1}$, more typical of rich groups. In the right panel of Figure 10, we compare the combined fractions of the two low-velocity dispersion clusters, $f_{\mathrm{A}}\left(M_{R}<-20, L_{\mathrm{X}, B}>10^{41}, \sigma<500\right)=0.100_{-0.043}^{+0.062}$, to the four higher velocity dispersion clusters, $f_{\mathrm{A}}\left(M_{R}<-20, L_{\mathrm{X}, B}>\right.$ $\left.10^{41}, \sigma>500\right)=0.026_{-0.008}^{+0.010}$. Using the binomial theorem to calculate the confidence intervals on the fractions (e.g., Gehrels 1986), we find that the probability the two above fractions overlap is very small, $\sim 0.3 \%$. However, this could overestimate the significance of the result as there are 15 different combinations of two cluster groups we could make from our cluster sample. Therefore, we conservatively estimate that the AGN fraction is higher in lower velocity dispersion clusters at the $\sim 95 \%$ confidence level. A similar trend appears in the Abell 901/902 supercluster Gilmour et al. (2007). There, X-ray AGNs prefer to be in regions with grouplike environments (mainly based on galaxy density), as compared to field-like or cluster-like environments. An anticorrelation between optical AGN fraction and velocity dispersion (Popesso \& Biviano 2006) and a correlation between radio AGN fraction and larger environmental densities (Best et al. 2005) have also been observed. We do not expect that this result is sensitive to the varying radial coverage between individual clusters. First, we found no radial dependence in the AGN fraction at these luminosities. Second, the discrepancy between the fractions is even more significant if we exclude Abell 644 and Abell 3128, the two clusters with the least complete radial coverage.

Smaller X-ray AGN fractions than that found in our low velocity dispersion clusters are measured in less dense environments. Shen et al. (2007) only found one X-ray AGN (out of $50 M_{R}<$ -20 galaxies) in a sample of eight $z \sim 0.06$ poor groups $(\sigma<$ $\left.500 \mathrm{~km} \mathrm{~s}^{-1}\right), f_{\mathrm{A}}\left(M_{R}<-20, L_{\mathrm{X}, B}>10^{41}\right)=0.020_{-0.017}^{+0.044}$, where the majority of these groups had smaller velocity dispersion than Abell 89B and Abell 3125. The X-ray AGN fraction of earlytype field galaxies in the Extended Chandra Deep Field-South has also been measured (Lehmer et al. 2007). They find $f_{\mathrm{A}}\left(M_{R}<\right.$ $\left.-20, L_{\mathrm{X}, B}>10^{41}\right)=0.066_{-0.024}^{+0.034}$ (B. Lehmer 2006, private communication). We note that the X-ray AGN fraction for all $M_{R}<$ -20 galaxies drops by a factor of $\sim 2$ compared to the fraction for just early-type galaxies in nearby clusters ( T. Arnold et al. 2008, in preparation). Since late-type galaxies are more prevalent in the field than in clusters, one expects the field X-ray AGN fraction for all galaxies to drop more rapidly compared to the $\sim 7 \%$ measured for early-type galaxies.

We note that any additional obscuration associated with gasrich galaxies will be more prevalent where the fraction of late-type galaxies is higher. The effect of missing AGNs due to obscuration will be strongest in the field and weakest in the highest velocity dispersion clusters. Thus, obscuration is unlikely to explain the apparent prevalence of X-ray AGNs in rich groups and poor clusters.

The likelihood of galaxy mergers increases with increasing galaxy density and decreasing relative velocity. Compared to poor groups and the field, the galaxy densities of rich groups are higher. Compared to galaxies in clusters, the relative velocities of galaxies in rich groups are lower. Thus, it is not surprising that AGNs may form preferentially in grouplike environments. A larger sample of groups and clusters, particularly those with velocity dispersions of poor clusters or rich groups at $\sigma \sim 500 \mathrm{~km} \mathrm{~s}^{-1}$, are needed to determine the preferred environment for AGNs and to use this information to determine how they are fueled. Larger data sets of comparably selected X-ray AGNs in the field would also be valuable.

\section{CONCLUSIONS}

To better understand the factors that may drive the evolution of AGNs today, we measure the AGN fraction in a new sample of nearby rich clusters, compare it to more distant samples, and examine how it varies with environment. We present new widefield Chandra observations of AGNs in Abell 85 and Abell 754. Seventeen X-ray sources associated with galaxies in Abell 85, Abell 89B, and Abell 754 are detected. Using $L_{\mathrm{X}} / L_{K_{s}}$ relations we classify seven of these galaxies as X-ray AGNs with $L_{\mathrm{X}, B}>$ $10^{41} \mathrm{ergs} \mathrm{s}^{-1}$. Only two of these X-ray AGNs are classified as AGNs based on their optical spectra. Two of the X-ray galaxies not classified as X-ray AGNs have been previously spectroscopically identified as AGNs. These sources are examples of the additional AGNs we expect are present in these clusters below our luminosity threshold.

We add detections of X-ray AGNs in three other $z \lesssim 0.08$ clusters, Abell 644, Abell 3125, and Abell 3128, to create a sample of 16 X-ray AGNs. We find that $3.4_{-0.8}^{+1.1} \%$ of $M_{R}<-20$ galaxies host X-ray AGNs with $L_{\mathrm{X}, B}>10^{41} \mathrm{ergs} \mathrm{s}^{-1}$. These results are consistent with the $\sim 5 \%$ fractions from M06 and M07. When compared to other samples (e.g., M07; Sun et al. 2007) at the same rest-frame X-ray luminosity and absolute magnitude limits, there is no evidence for an evolving X-ray AGN fraction for $z \lesssim 0.3$. 
Our three most important results are as follows:

1. X-ray AGNs are detected more often in more luminous host galaxies. This observed trend of X-ray AGN fraction highlights the importance of using the same X-ray luminosity and absolute magnitude cuts when comparing fractions. This trend is due at least partially to a physical selection effect. Two galaxies can have the same accretion rate relative to the Eddington limit, but the galaxy with the more massive black hole will have a higher $\mathrm{X}$-ray luminosity. More luminous host galaxies tend to have more massive black holes for a combination of two reasons. First, the mass of a black hole scales with the mass of a bulge component, and at a given bulge-to-disk ratio, a more luminous galaxy will have a more massive bulge. Second, more luminous host galaxies are more likely to be dominated by their spheroid. Thus, it is not surprising that at a given X-ray luminosity one can detect less efficient X-ray AGNs in more luminous galaxies. We require an understanding of the distribution of accretion rates relative to the Eddington limit in X-ray AGNs to determine whether X-ray AGNs are more likely to reside in more luminous host galaxies for reasons beyond this physical selection effect.

2. We do not find an excess fraction of $X$-ray $A G N$ s in the outskirts of clusters. The radial distribution of X-ray AGNs appears to follow the same distribution as cluster members without X-ray AGNs. Ruderman \& Ebeling (2005) also found a relatively flat distribution of X-ray sources around massive, disturbed clusters. The expectation from the pictures in which galaxy-galaxy encounters (close tidal interactions and major mergers) or galaxy harassment fuel AGNs is that more AGNs should be found in the outskirts of clusters. Acting against this expectation is our increased sensitivity to AGNs in the most luminous spheroids, as well as the significant fraction of early-type galaxies known to harbor low-luminosity AGNs and LINERs. Thus, the tendency of the most massive and early-type galaxies to lie in the centers of richest clusters could dilute or even reverse AGN trends due to galaxy interactions. For instance, Ruderman \& Ebeling (2005) also found that massive, relaxed clusters had an excess number of X-ray AGNs in their central $0.5 \mathrm{Mpc}$ and near their virial radius. The lack of any trend in our results could arise from the combination of effects listed above or from radial averaging over the substructure in the cluster, or it could be because there is actually no preferred radial distribution for X-ray AGNs.

3. There is increasing evidence for higher AGN fractions in low-velocity dispersion clusters. We find that the fraction of $\mathrm{X}$-ray AGNs is larger (at the $\sim 95 \%$ confidence level) in lower velocity dispersion clusters or large groups $\left(10.0_{-4.3}^{+6.2} \%\right)$ than in richer clusters $\left(2.6_{-0.8}^{+1.0} \%\right)$. Combining results for the Abell $901 / 902$ supercluster (Gilmour et al. 2007), poor groups (Shen et al. 2007), and the field (Lehmer et al. 2007), one can form a picture where
X-ray AGNs in the local universe are preferentially found in rich group environments. If gas-rich mergers between galaxies are the principal driver of AGNs, then there should be more AGNs in groups where the galaxies tend to have higher gas fractions and smaller relative velocities than in richer clusters, while galaxy densities are higher than in the field. Because the most massive and early-type galaxies tend to lie in the richest clusters, and these galaxies often harbor detectable LINERs that may be lowluminosity AGNs, any increase in AGN fraction due to galaxygalaxy interactions could be even larger than what we measure. Finally, we note that obscuration of X-ray AGNs in gas-rich galaxies is not likely to be responsible for this trend, as more gasrich galaxies are expected in lower density environments.

These issues can be addressed through studies of larger samples of X-ray AGNs for clusters, groups, and the field. For clusters, there are a couple of key properties that need to be better sampled: an increased number of lower velocity dispersion clusters and a larger number of disturbed and relaxed clusters observed out to their virial radius. Such samples would directly address the radial distribution of X-ray AGNs and whether there is a preferred host environment. Specifically, such data would be valuable to resolve the potential inconsistency between our second and third highlighted results, which may be due to small number statistics in the outskirts of clusters or our averaging over the substructure in clusters. In addition, larger numbers of X-ray AGNs will constrain the underlying Eddington accretion efficiency distribution and (host luminosity dependent?) AGN fraction by convolving these properties with the galaxy luminosity function and comparing to the observed AGN fractions. These constraints in turn can be tested against AGN fueling mechanisms.

We thank Francesco Shankar for a useful discussion on accretion efficiency. Support for this work was provided by the National Aeronautics and Space Administration (NASA) through Chandra awards GO4-5122A and GO6-7091X, issued by the Chandra X-Ray Observatory, which is operated by the Smithsonian Astrophysical Observatory for and on behalf of NASA under contract NAS8-03060. This research has made use of the NASA/ IPAC Extragalactic Database (NED), which is operated by the Jet Propulsion Laboratory, California Institute of Technology, under contract with NASA. The Second Palomar Observatory Sky Survey (POSS-II) was made by the California Institute of Technology with funds from the National Science Foundation, the National Geographic Society, the Sloan Foundation, the Samuel Oschin Foundation, and the Eastman Kodak Corporation.

Facilities: CXO (ACIS)
Adelman-McCarthy, J. K., et al. 2007, ApJS, 172, 634

Barbaro, G., \& Poggianti, B. M. 1997, A\&A, 324, 490

Barnes, J. E., \& Hernquist, L. 1992, ARA\&A, 30, 705

Beers, T. C., Flynn, K., \& Gebhardt, K. 1990, AJ, 100, 32

Best, P. N., Kauffmann, G., Heckman, T. M., Brinchmann, J., Charlot, S., Ivezić, Ž., \& White, S. D. M. 2005, MNRAS, 362, 25

Bruzual, G., \& Charlot, S. 2003, MNRAS, 344, 1000

Caldwell, N., \& Rose, J. A. 1997, AJ, 113, 492

Calzetti, D., Armus, L., Bohlin, R. C., Kinney, A. L., Koornneef, J., \& Storchi-

Bergmann, T. 2000, ApJ, 533, 682

Chabrier, G. 2003, ApJ, 586, L133

Christlein, D., \& Zabludoff, A. I. 2003, ApJ, 591, 764 (CZ03) 2005, ApJ, 621, 201

Condon, J. J. 1992, ARA\&A, 30, 575

Condon, J. J., Anderson, M. L., \& Helou, G. 1991, ApJ, 376, 95

\section{REFERENCES}

Condon, J. J., Cotton, W. D., Greisen, E. W., Yin, Q. F., Perley, R. A., Taylor, G. B., \& Broderick, J. J. 1998, AJ, 115, 1693

Davis, J. E. 2001, ApJ, 562, 575

Dressler, A. 1980, ApJ, 236, 351

Dressler, A., Thompson, I. B., \& Shectman, S. A. 1985, ApJ, 288, 481

Durret, F., Forman, W., Gerbal, D., Jones, C., \& Vikhlinin, A. 1998, A\&A, 335, 41

Durret, F., Lima Neto, G. B., \& Forman, W. 2005, A\&A, 432, 809

Eastman, J., Martini, P., Sivakoff, G., Kelson, D. D., Mulchaey, J. S., \& Tran, K.-V. 2007, ApJ, 664, L9

Fisher, D., Fabricant, D., Franx, M., \& van Dokkum, P. 1998, ApJ, 498, 195 Gehrels, N. 1986, ApJ, 303, 336

Gilmour, R., Gray, M. E., Almaini, O., Best, P., Wolf, C., Meisenheimer, K., Papovich, C., \& Bell, E. 2007, MNRAS, 380, 1467

Giovanelli, R., \& Haynes, M. P. 1985, ApJ, 292, 404 
Gisler, G. R. 1978, MNRAS, 183, 633

Grimm, H.-J., Gilfanov, M., \& Sunyaev, R. 2003, MNRAS, 339, 793

Hewitt, A., \& Burbidge, G. 1991, ApJS, 75, 297

Ho, L. C., Filippenko, A. V., \& Sargent, W. L. W. 1993, ApJ, 417, 63 1997, ApJ, 487, 568

Hornschemeier, A. E., Heckman, T. M., Ptak, A. F., Tremonti, C. A., \& Colbert, E. J. M. 2005, AJ, 129, 86

Katgert, P., Mazure, A., den Hartog, R., Adami, C., Biviano, A., \& Perea, J. 1998, A\&AS, 129, 399

Kauffmann, G., et al. 2003a, MNRAS, 341, 54 . 2003b, MNRAS, 346, 1055

Kempner, J. C., Sarazin, C. L., \& Ricker, P. M. 2002, ApJ, 579, 236

Kennicutt, R. C., Jr. 1992, ApJ, 388, 310 . 1998, ARA\&A, 36, 189

Kim, D., \& Fabbiano, G. 2003, ApJ, 586, 826 2004, ApJ, 611, 846

Kim, M., et al. 2007, ApJS, 169, 401

Lake, G., Katz, N., \& Moore, B. 1998, ApJ, 495, 152

Lamareille, F., Mouhcine, M., Contini, T., Lewis, I., \& Maddox, S. 2004, MNRAS, 350, 396

Lauberts, A., \& Valentijn, E. A. 1989, The Surface Photometry Catalogue of the ESO-Uppsala Galaxies (Garching: ESO)

Lehmer, B. D., et al. 2007, ApJ, 657, 681

Majewski, S. R., Skrutskie, M. F., Weinberg, M. D., \& Ostheimer, J. C. 2003, ApJ, 599, 1082

Marconi, A., \& Hunt, L. K. 2003, ApJ, 589, L21

Markevitch, M., et al. 2003, ApJ, 586, L19

Marshall, H. L., Miller, B. P., Davis, D. S., Perlman, E. S., Wise, M., Canizares, C. R., \& Harris, D. E. 2002, ApJ, 564, 683

Martini, P., Kelson, D. D., Kim, E., Mulchaey, J. S., \& Athey, A. A. 2006, ApJ, 644, 116 (M06)

Martini, P., Kelson, D. D., Mulchaey, J. S., \& Trager, S. C. 2002, ApJ, 576, L109

Martini, P., Mulchaey, J. S., \& Kelson, D. D. 2007, ApJ, 664, 761 (M07)
Matt, G. 2002, Philos. Trans. R. Soc. London A, 360, 2045

McLure, R. J., \& Dunlop, J. S. 2002, MNRAS, 331, 795

Moore, B., Katz, N., Lake, G., Dressler, A., \& Oemler, A. 1996, Nature, 379, 613

Moran, E. C., Filippenko, A. V., \& Chornock, R. 2002, ApJ, 579, L71

Moshir, M., et al. 1990, IRAS Faint Source Catalog (ver. 2.0; Pasadena: IPAC) Paturel, G., Petit, C., Prugniel, P., Theureau, G., Rousseau, J., Brouty, M.,

Dubois, P., \& Cambrésy, L. 2003, A\&A, 412, 45

Popesso, P., \& Biviano, A. 2006, A\&A, 460, L23

Press, W. H., Teukolsky, S. A., Vetterling, W. T., \& Flannery, B. P. 1992, Numerical Recipes in C. The Art of Scientific Computing (2nd ed.; Cambridge: Cambridge Univ. Press)

Puchnarewicz, E. M., et al. 1992, MNRAS, 256, 589

Rola, C. S., Terlevich, E., \& Terlevich, R. J. 1997, MNRAS, 289, 419

Ruderman, J. T., \& Ebeling, H. 2005, ApJ, 623, L81

Schlegel, D. J., Finkbeiner, D. P., \& Davis, M. 1998, ApJ, 500, 525

Shen, Y., Mulchaey, J. S., Raychaudhury, S., Rasmussen, J., \& Ponman, T. J. 2007, ApJ, 654, L115

Sivakoff, G. R., Sarazin, C. L., \& Irwin, J. A. 2003, ApJ, 599, 218 2004, ApJ, 617, 262

Skrutskie, M. F., et al. 2006, AJ, 131, 1163

Sun, M., Jones, C., Forman, W., Vikhlinin, A., Donahue, M., \& Voit, M. 2007, ApJ, 657, 197

Thomas, T., \& Katgert, P. 2006, A\&A, 446, 31

Treu, T., Ellis, R. S., Kneib, J.-P., Dressler, A., Smail, I., Czoske, O., Oemler, A., \& Natarajan, P. 2003, ApJ, 591, 53

Ueda, Y., Akiyama, M., Ohta, K., \& Miyaji, T. 2003, ApJ, 598, 886

Vasudevan, R. V., \& Fabian, A. C. 2007, MNRAS, 381, 1235

White, R. L., Becker, R. H., Helfand, D. J., \& Gregg, M. D. 1997, ApJ, 475, 479

Yuan, F., \& Narayan, R. 2004, ApJ, 612, 724

Yun, M. S., Reddy, N. A., \& Condon, J. J. 2001, ApJ, 554, 803

Zabludoff, A. I., \& Zaritsky, D. 1995, ApJ, 447, L21

Zhao, J.-H., Burns, J. O., \& Owen, F. N. 1989, AJ, 98, 64 